\title{
OBSERVING THE UNOBSERVABLE? MODELING CORONAL CAVITY DENSITIES
}

\author{
J. Fuller, ${ }^{1}$ S. E. Gibson, ${ }^{2}$ G. DE Toma, ${ }^{2}$ And Y. FAN ${ }^{2}$ \\ Received 2007 November 14; accepted 2008 January 22
}

\begin{abstract}
Prominence cavities in coronal helmet streamers are readily detectable in white-light coronagraph images, yet their interpretation may be complicated by projection effects. In order to determine a cavity's density structure, it is essential to quantify the contribution of noncavity features along the line of sight. We model the coronal cavity as an axisymmetric torus that encircles the Sun at constant latitude and fit it to observations of a white-light cavity observed by the Mauna Loa Solar Observatory (MLSO) MK4 coronagraph from 2006 January 25 to 30. We demonstrate that spurious noncavity contributions (including departures from axisymmetry) are minimal enough to be incorporated in a density analysis as conservatively estimated uncertainties in the data. We calculate a radial density profile for cavity material and for the surrounding helmet streamer (which we refer to as the "cavity rim") and find that the cavity density is depleted by a maximum of $40 \%$ compared to the surrounding helmet streamer at low altitudes $\left(1.18 R_{\odot}\right)$ but is consistently higher (double or more) than in coronal holes. We also find that the relative density depletion between cavity and surrounding helmet decreases as a function of height. We show that both increased temperature in the cavity relative to the surrounding helmet streamer and a magnetic flux rope configuration might lead to such a flattened density profile. Finally, our model provides general observational guidelines that can be used to determine when a cavity is sufficiently unobstructed to be a good candidate for plasma diagnostics.
\end{abstract}

Subject headings: Sun: corona - Sun: coronal mass ejections (CMEs) — Sun: filaments — Sun: magnetic fields — Sun: prominences

Online material: color figures

\section{INTRODUCTION}

The complexity of the solar corona continues to be demonstrated by ever more sophisticated observations that probe coronal structure and dynamics to higher and higher spatial and temporal resolution. Such complexity can be overwhelming, so it is useful to consider simpler, large-scale, long-lived structures, which may illuminate the underlying order of the solar atmosphere. Coronal cavities are examples of such structures. These dark, semicircular or circular regions often surround a central prominence and may be embedded in helmet streamers (Fig. 1). They provide clues to magnetohydrodynamic equilibrium states of the solar corona and to the processes that can destabilize these equilibria and drive coronal mass ejections (CMEs).

Coronal cavities have been studied for decades (see TandbergHanssen 1974, 1995; Engvold 1989; Gibson et al. 2006, for reviews). They are the limb equivalent of filament channels and so are ubiquitous. They come in all sizes, from cavities associated with large-scale, longitudinally extended polar crown filaments to smaller ones associated with active regions. Cavities of large radial extent are visible in white-light occulted coronagraph observations (e.g., Figs. 1 and 2), while those that do not extend as high up are better observed in unocculted observations such as extreme ultraviolet (EUV) or soft X-ray (SXR) emission. Cavities have been demonstrated to erupt as CMEs, in some cases exhibiting a slow rise and increase in cavity contrast prior to eruption (Fisher \& Poland 1981; Illing \& Hundhausen 1986; Hundhausen 1999; Yurchyshyn 2002; Maričić et al. 2004; Sterling \& Moore 2004; Vršnak et al. 2004; Gibson et al. 2006).

Because cavities are dark in white-light coronal observations, which depend on coronal electron density without sensitivity to

\footnotetext{
${ }^{1}$ Whitman College, 280 Boyer Avenue, Walla Walla, WA 99362; c/o S. Gibson, HAO/NCAR, P.O. Box 3000, Boulder, CO 80307-3000.

2 HAO/NCAR, P.O. Box 3000, Boulder, CO 80307-3000.
}

temperature structure, it is clear that they are regions of depleted density. Analysis of white-light and radio cavity observations have yielded estimates of cavity density depletion ranging from $20 \%$ to $80 \%$ (Marque 2004; Kundu et al. 1978; Saito \& Hyder 1968; Saito \& Tandberg-Hanssen 1973; Straka et al. 1975). Gibson et al. (2006) presented a survey of 75 days of cavity observations, finding a range in white-light intensity ( polarized brightness, or $p B$ ) poleward depletion of $14 \%-43 \%$. It is important to note that despite this depletion, the intensity in the cavity is still significantly greater than in surrounding coronal holes (see Fig. 3, top).

The trouble with determining density from white-light cavity observations lies in the fact that any image is necessarily the twodimensional projection of a three-dimensional structure. Because the corona is optically thin, the intensity of each pixel in an image represents the integration of all the contributions along the line of sight (albeit multiplied by a scattering function, which falls off with height). Observing the cavity without contamination from surrounding features can prove to be difficult, since cavities are usually surrounded by bright helmet streamers, and unrelated structures such as active regions or coronal holes may also lie along the line of sight. EUV observations of cavities along multiple lines of sight as obtained by the STEREO satellite will help disentangle such three-dimensional effects. However, we can also make progress using simple geometrical arguments under optimal cavity-viewing conditions.

In many cases cavities exist as long-term structures that maintain roughly the same size and position for days or even weeks at a time (Gibson et al. 2006). Since features in the Sun's corona rotate at about $13.5^{\circ}$ day $^{-1}$, the seemingly constant location of cavities on the limb of the Sun implies that many cavities have a large longitudinal extent and actually wrap around a significant portion of the Sun. In other words, cavities are shaped like tunnels through the corona. If a cavity maintains constant latitude on the limb of the Sun for several days, the cavity can be envisioned as 

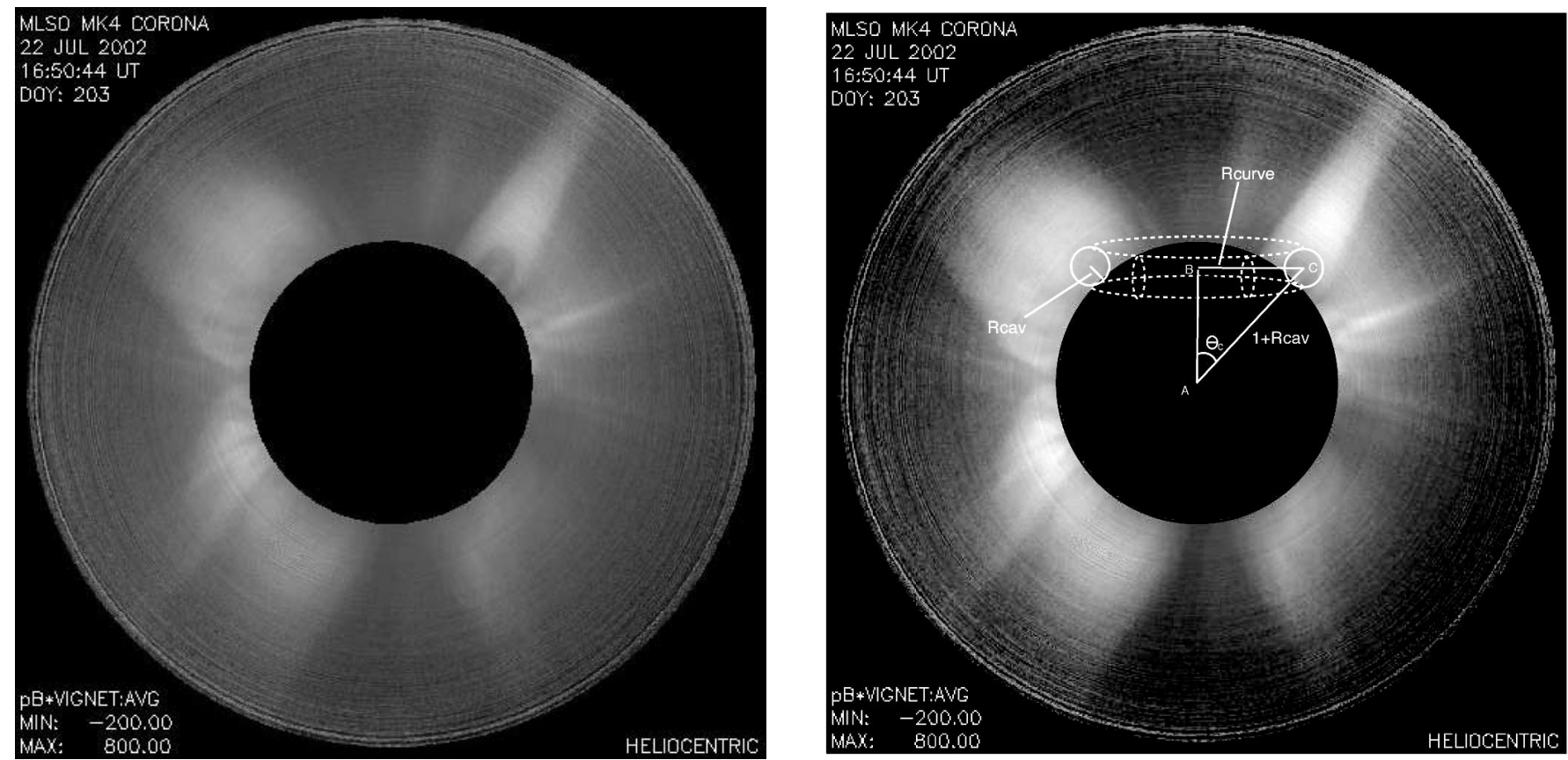

FIG. 1. - Left: Coronal cavity present in the northwest quadrant on 2002 July 22, as observed by the Mauna Loa Solar Observatory (MLSO) MK4 white-light coronameter. Right: With model diagram overlaid. See text and Figs. 5 and 6 for explanation of model variables.

an axisymmetric feature that encircles the Sun at constant latitude (Fig. 1). As we will show in this paper, in this scenario the primary contamination to a pure cavity signal will come from the projection of bright material lying above the tunnel as it bends into the line of sight. Under the right circumstances, even this contribution is minimal and we can safely say that we are observing primarily cavity material.

In $\oint 2$ of this paper, we describe our model for the cavity and discuss its geometrical implications. In $\S 3$, we fit observations of the white-light cavity of 2006 January 25-30 (Figs. 2-3) with

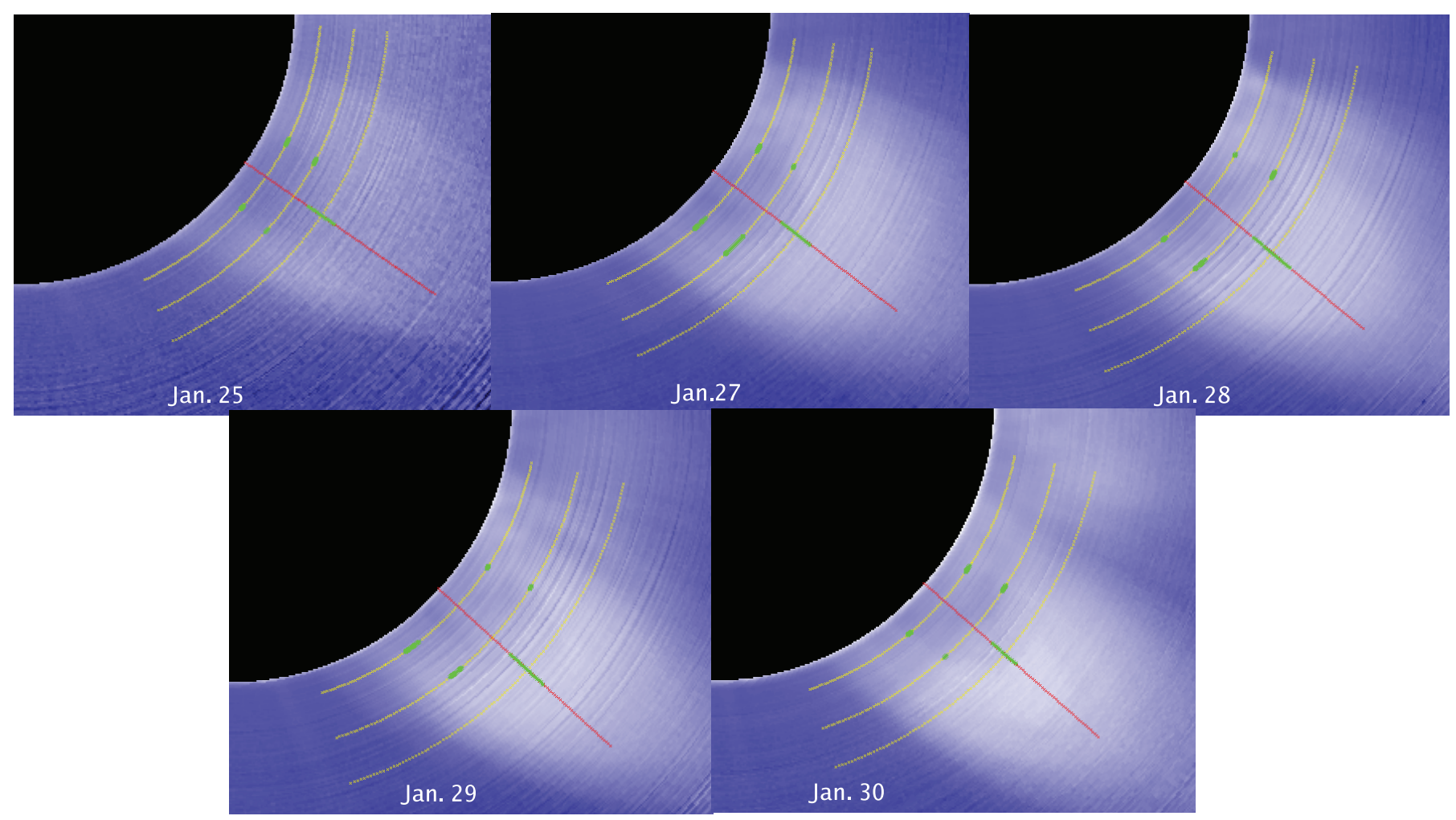

FIG. 2. - Observations from MLSO/MK4 of the coronal cavity that existed from 2006 January 25 until January 30 in the SW coronal quadrant (between approximately $124^{\circ}$ and $132^{\circ}$ polar angle). A radial gradient function has been applied to these images to enhance contrast. No data were available for January 26 , but it is reasonable to assume that the cavity was present on this day as well. The red lines show the central angle $\theta_{c}$. The yellow lines show three heights, including $r=1.2$, the height of the top of the cavity, and halfway in between. The green asterisks indicate the edge of the cavity (rim/cavity interface; see Figs. 4 and 5) with a spread indicating uncertainty. See Gibson et al. (2006) for further description of the cavity-fitting process. 

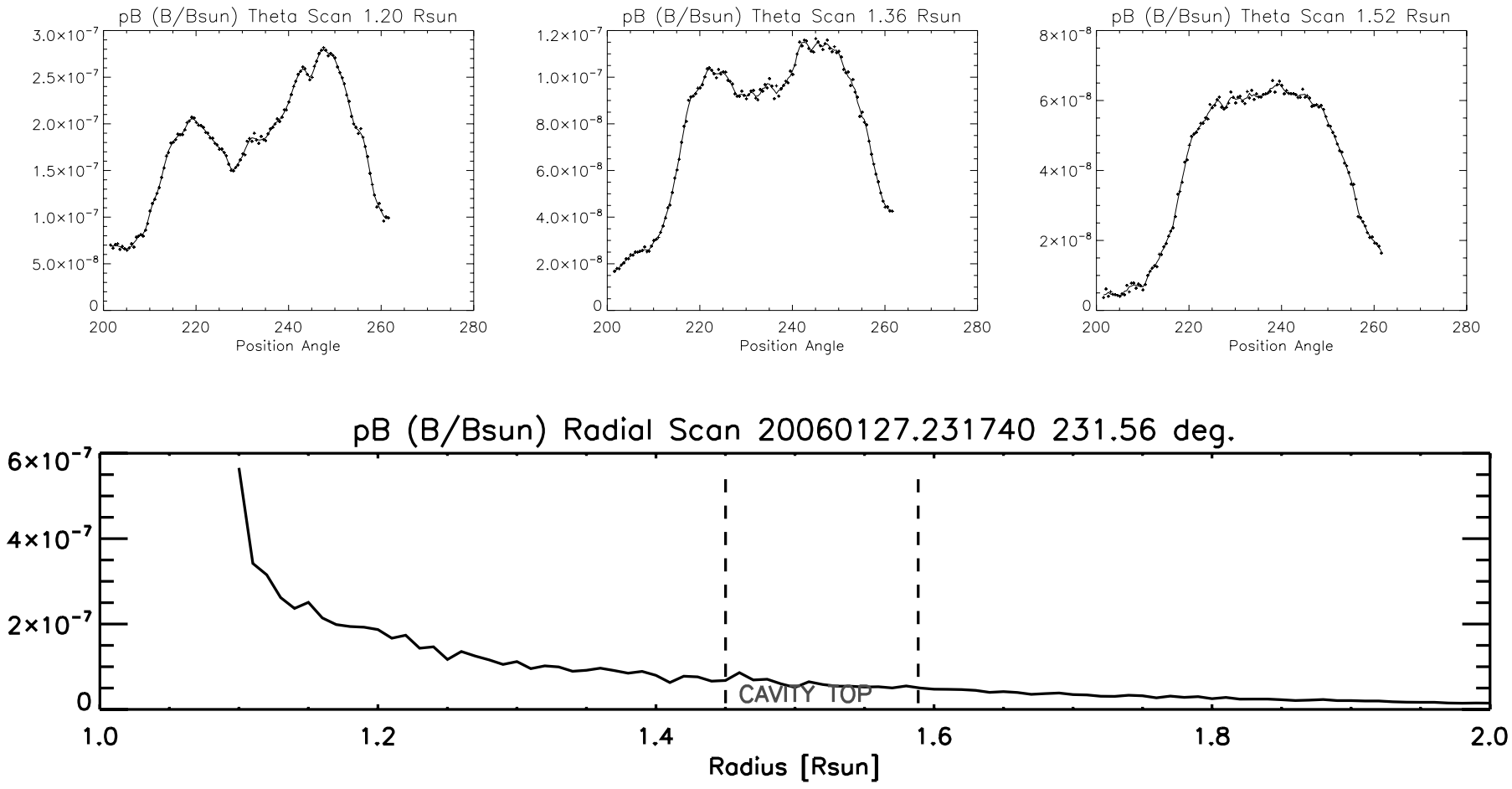

FIG. 3.-Top: Polarized brightness $(p B)$ at constant altitude as a function of position angle (measured counterclockwise from the north pole) as measured on 2006 January 27 , by the MLSO/MK4 coronameter. The January 27 cavity is represented in the latitudinal scans by the trough between the two peaks, which are created by the surrounding helmet streamer. Note that even the dimmest part of the cavity at $1.2 R_{\odot}$ is substantially brighter than the coronal holes that lie on either side of the helmet streamer. Bottom: $p B$ as a function of radial distance away from the Sun in the plane of the sky. The helmet streamer around the top of the cavity is dimmer than most if not all of the cavity, so there is no distinct cavity boundary identifiable in this central radial profile. The location of the cavity top is better determined as the height where the trough vanishes in the latitudinal scans (e.g., top right), or using contrast-enhanced data (e.g., Fig. 2). The dashed lines in the bottom scan indicate the uncertainty in this cavity top position (also illustrated by the green asterisks in Fig. 2). [See the electronic edition of the Journal for a color version of this figure.]

our model. In $\S 4$ we determine the radial density profiles of the cavity versus surrounding helmet and discuss uncertainties. In $\S 5$, we discuss implications for cavity temperature and magnetic field structure. In $\S 6$, we present our conclusions.

\section{MODEL OF THE CAVITY}

As discussed above, the cavity can be modeled as an axisymmetric torus that encircles the Sun at constant colatitude $\theta_{c}$ (Figs. 1, 4, and 5). We model the surrounding streamer also as

\section{AXISYMMETRIC CAVITY}

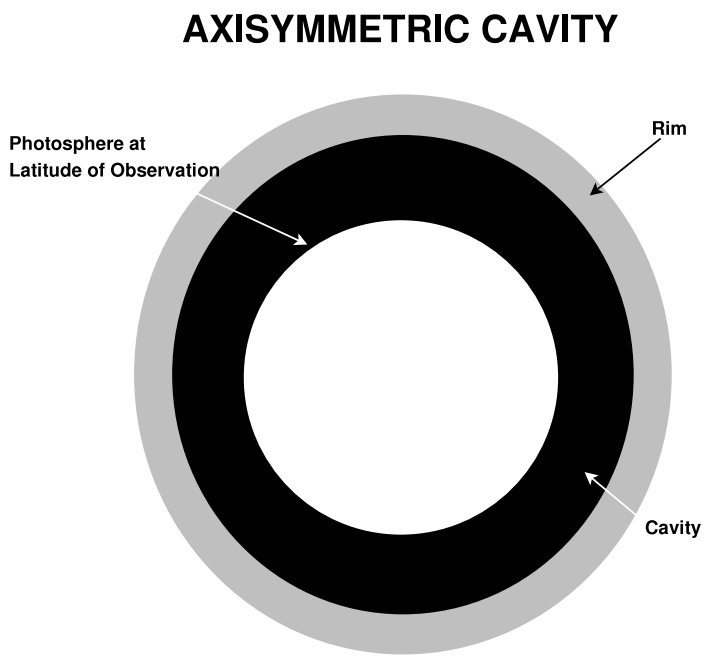

an axisymmetric torus, centered at the same point as the cavity torus and truncated at the photosphere (Figs. 4 and 5). As Figures 1 and 2 show, a real streamer is more radially extended than this, and we will explicitly include this additional contribution of the surrounding streamer to the line-of-sight integral in our calculations ( $(4.1)$. However, the concept of a toroidal "cavity rim" having a width consistent with the lateral brightenings on either side of the cavity is useful for understanding the geometry of projected structures. Real cavities are also likely not to be completely

\section{ASYMMETRIC CAVITY}

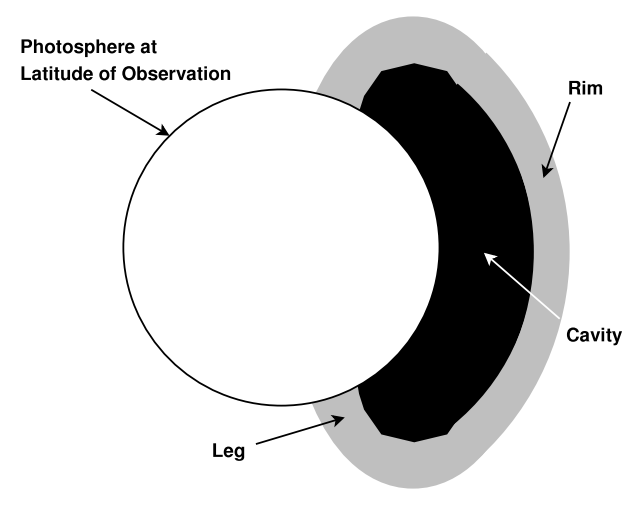

FIG. 4. - View looking down from north pole of a schematic representation of an axisymmetric cavity/rim system (left), and an asymmetric cavity/rim/leg system (right). The $x y$-plane shown would be at the latitude where the cavity touches the solar photosphere. 

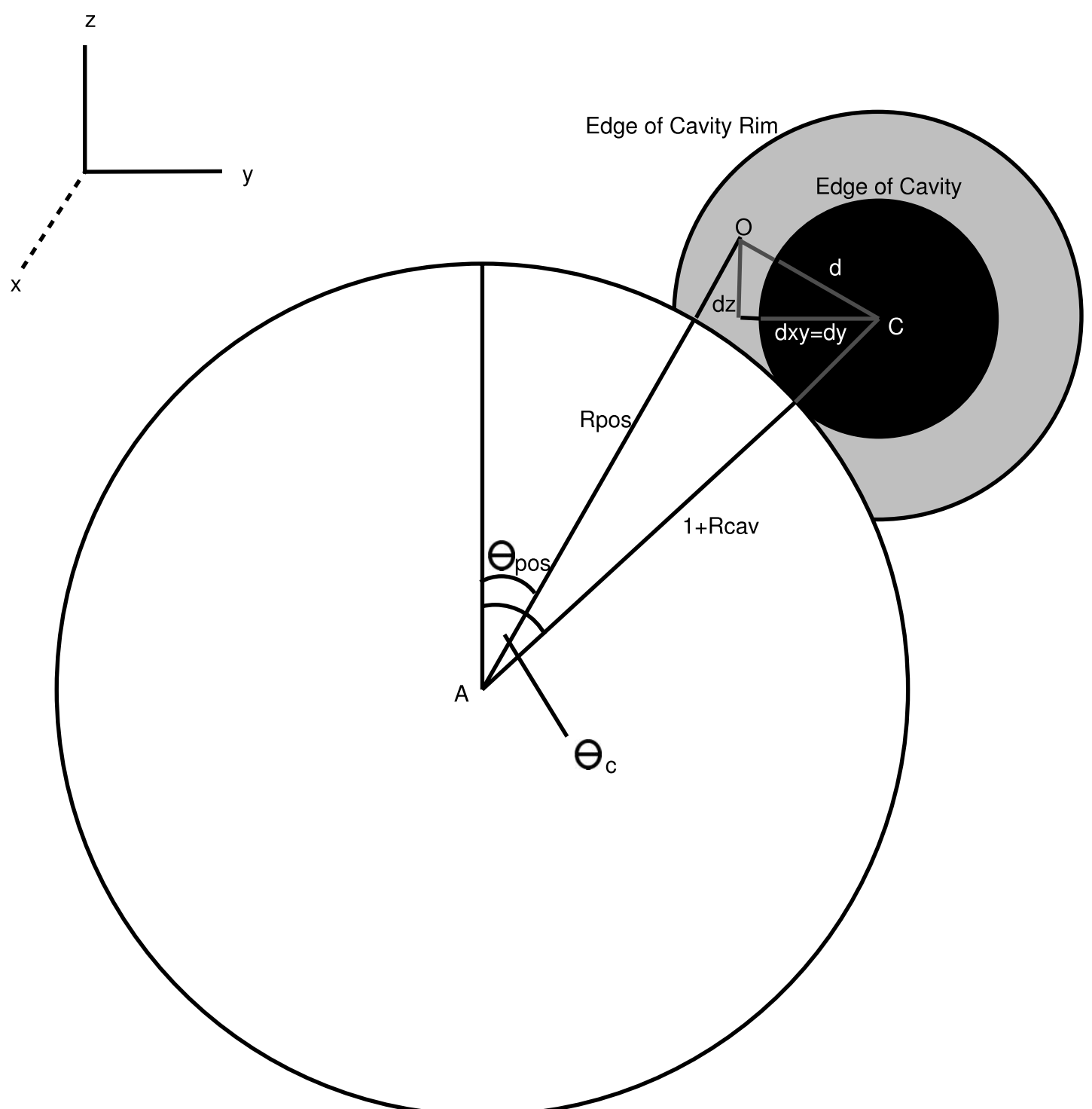

FIG. 5.- Sun and axisymmetric cavity/rim system as viewed in the plane of the sky. Point $\mathrm{O}$ is the point of observation (in the case pictured, it lies in the cavity rim) and has polar coordinates $\left(R_{\mathrm{pos}}, \theta_{\mathrm{pos}}\right)$. Point $\mathrm{C}$ is the center of the cavity in the plane of the sky. The variable $d$ is the distance from the cavity center at a given colongitude to the point along O's line of sight at the same colongitude (see also Fig. 6). This can be decomposed into a component in the $x y$-plane, $d_{x y}$, and a component perpendicular to this plane, $d_{z}$. In the plane of the sky (colongitude $\phi=0$ ) as shown in this figure, $d_{x y}$ is purely in the $y$-direction. The distance $d_{z}$ is constant for all points along O's line of sight, but the distance $d_{x y}$ changes with longitude.

axisymmetric but rather to have rims that bend down to the photosphere (Fig. 4), which we will refer to as "cavity legs." We discuss this further in $\S 2.2$, but to begin with, we will consider what can be learned by assuming axisymmetry.

Let us describe the variables used in this model, as shown in Figures 5-6. We can easily measure the radius $R_{\text {cav }}$ and colatitude $\theta_{c}$ of any cavity by simply examining white-light images. Our geometry utilizes distances in units of solar radii $\left(R_{\odot}\right)$, and we will use these units throughout the paper. The distance from the center of the Sun to a point of observation $\mathrm{O}$ in the plane of the sky is $R_{\text {pos }}$, and the colatitude of $\mathrm{O}$ is $\theta_{\text {pos }}$. The variable $R_{\text {curve }}$ is the radius of curvature of the cavity and is defined as $R_{\text {curve }}=$ $\left(1+R_{\text {cav }}\right) \sin \theta_{c}$. The variable $R_{\phi}$ is the distance in the $x y$-plane between a point along O's line of sight and the Sun's axis of rotation. The line-of-sight angle $\alpha$, which will later be referred to as the scattering angle for white light, is a variable that determines which point along a line of sight we are examining. The angle $\phi$, which we measure from the limb of the Sun rather than the central meridian and so refer to as a "colongitude," can also be used to specify a point along the line of sight. At the equator $\alpha=\phi$, and in general the two can be related using the equation

$$
\tan \alpha=\tan \phi \sin \theta_{\text {pos }} \text {. }
$$

The distance from the center of the Sun of any point D along O's line of sight at a given line-of-sight angle is $R_{\text {plasma }}$, corresponding to the line segment AD in Figure 6, and is given by the equation

$$
R_{\text {plasma }}=R_{\text {pos }} / \cos \alpha .
$$

As Figures 5 and 6 show, the distance $d$ from the point D to the cavity center at D's colongitude can be decomposed into a component in the $x y$-plane, $d_{x y}$, which varies along the line of 


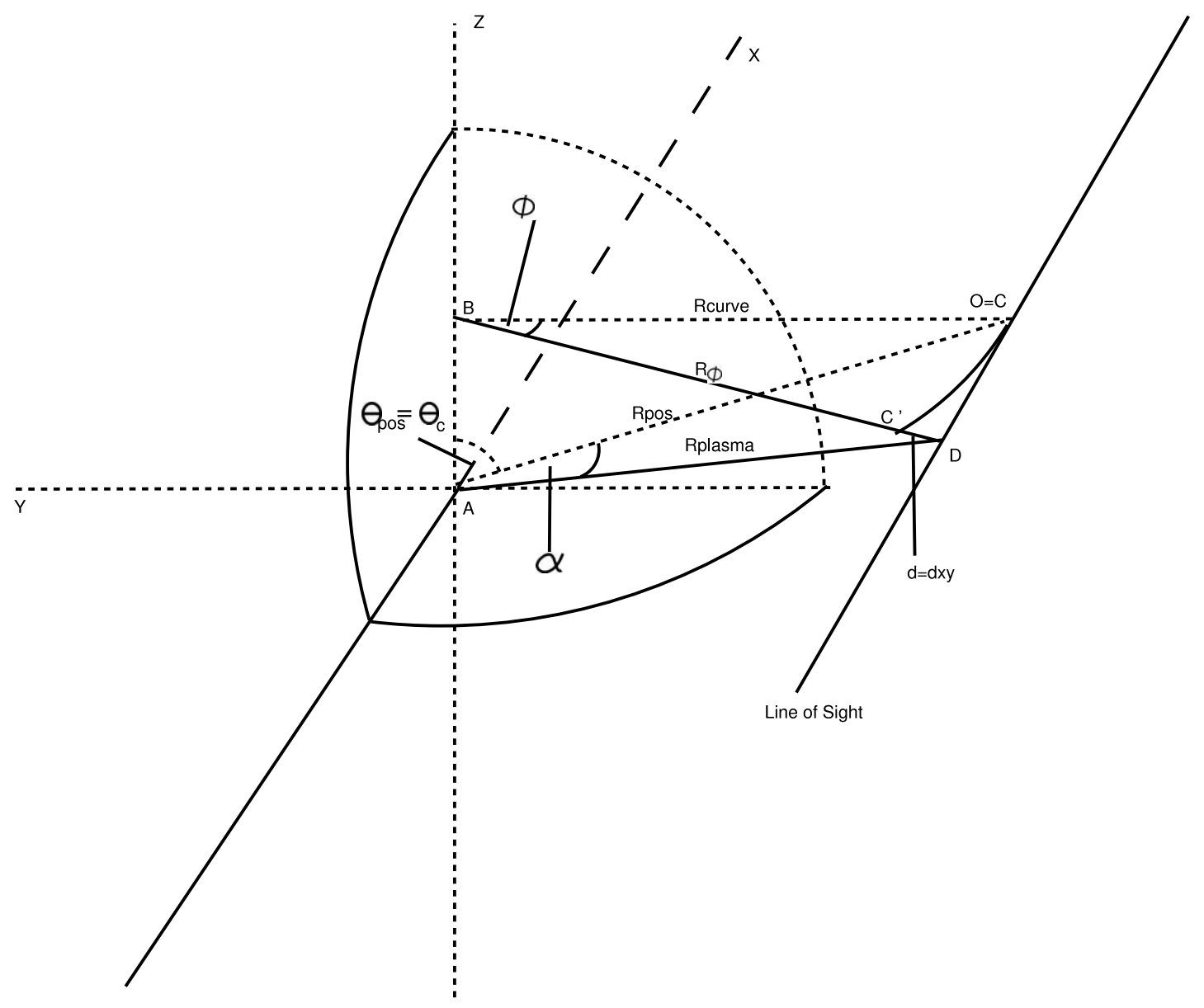

FIG. 6.- Geometry of the model. Point A lies at the center of the Sun, point B lies at the center of rotation of the cavity, point C is at the center of the cavity in the plane of the sky where its line of sight passes closest to the Sun, and point D lies at a line-of-sight angle $\alpha$ and a colongitude $\phi$. In this figure, the line of sight passes through the center of the cavity so that points $\mathrm{O}$ and $\mathrm{C}$ are identical. Points $\mathrm{A}, \mathrm{B}$, and $\mathrm{C}$ lie in the plane of sky, while points $\mathrm{B}, \mathrm{C}$, and $\mathrm{D}$ lie in the plane of rotation of the cavity. Triangle $\mathrm{BCD}$ is a projection of triangle $\mathrm{ACD}$ into the plane of rotation, while triangle $\mathrm{ABC}$ is a projection of triangle $\mathrm{ABD}$ into the plane of the sky. Solid lines are meant to project in the $x$-direction, while the dotted lines are confined to the $y z$-plane. The distance $d$ between the cavity center at colongitude $\phi\left(\mathrm{e} . \mathrm{g}\right.$., the point $\left.\mathrm{C}^{\prime}\right)$ and the point $\mathrm{D}$ is $d=d_{x y}\left(d_{z}=0\right)$. Note how the distance $d_{x y}$ will increase as we move further from the plane of the sky along the line of sight.

sight, and a component perpendicular to this plane, $d_{z}$, which remains constant for any colongitude. In particular, we find that

$d_{x y}=R_{\phi}-R_{\text {curve }}=R_{\text {pos }} \sqrt{\sin ^{2} \theta_{\text {pos }}+\tan ^{2} \alpha}-\left(1+R_{\text {cav }}\right) \sin \theta_{c}$,

$$
\begin{gathered}
d_{z}=R_{\mathrm{pos}} \cos \theta_{\mathrm{pos}}-\left(1+R_{\mathrm{cav}}\right) \cos \theta_{c}, \\
d=\sqrt{d_{x y}^{2}+d_{z}^{2}} .
\end{gathered}
$$

It will be useful to describe the scattering angle $\alpha$ along a line of sight in the following form:

$$
\alpha= \pm \arctan \sqrt{\left[\frac{\sqrt{d^{2}-d_{z}^{2}} \pm\left(1+R_{\mathrm{cav}}\right) \sin \theta_{c}}{R_{\mathrm{pos}}}\right]^{2}-\sin ^{2} \theta_{\mathrm{pos}}}
$$

\subsection{Projection Effects of the Cavity Rim}

White-light intensity observations include contributions from all along the line of sight, at a variety of angles and heights. Using equations (1) and (2) we can calculate the angle (either line-ofsight angle $\alpha$ or colongitude $\phi$ ) at which material from a given height enters a line of sight. Figure 7 demonstrates how material at heights greater than $R_{\text {pos }}$ enter into its line of sight as a function of $\alpha$ or $\phi$. Material from below $R_{\text {pos }}$ never enters into its line of sight.

For the purposes of our analysis, it is important to determine the critical angles at which the cavity rim projects onto the cavity. If the total distance $d$ is greater than the cavity radius, then we are no longer inside the cavity. By setting $d$ equal to $R_{\text {cav }}$ and solving for $\alpha$, we can determine the values of $\alpha$ that correspond to where the edge of the cavity enters into the line of sight. We will refer to these angles as $\alpha_{\text {cav }}$. From equation (6), we see that for a given line of sight, $\alpha_{\text {cav }}$ can have up to four values. If we choose a point $\mathrm{O}$ within the cavity in the plane of the sky, we will obtain two values of $\alpha_{\text {cav }}$ representing the angles at which the line of sight exits the cavity on either side of the plane of the sky. If our point $\mathrm{O}$ lies in the cavity rim in the plane of the sky, particularly at low heights, it is possible to have two values of $\alpha_{\text {cav }}$ at positive colongitudes and two values at negative colongitudes, as the line of sight leaves the rim and then enters and then exits a portion of the cavity on either side of the plane of the sky.

We can also calculate the values of $\alpha$ corresponding to the outer edge of the cavity rim by setting $d$ equal to $R_{\text {rim }}$, where $R_{\text {rim }}$ is the radius of the torus that represents the surrounding helmet streamer (see Fig. 5). Since we will never choose a point $O$ that does not lie in the cavity or the cavity rim in the plane of the sky, we will 

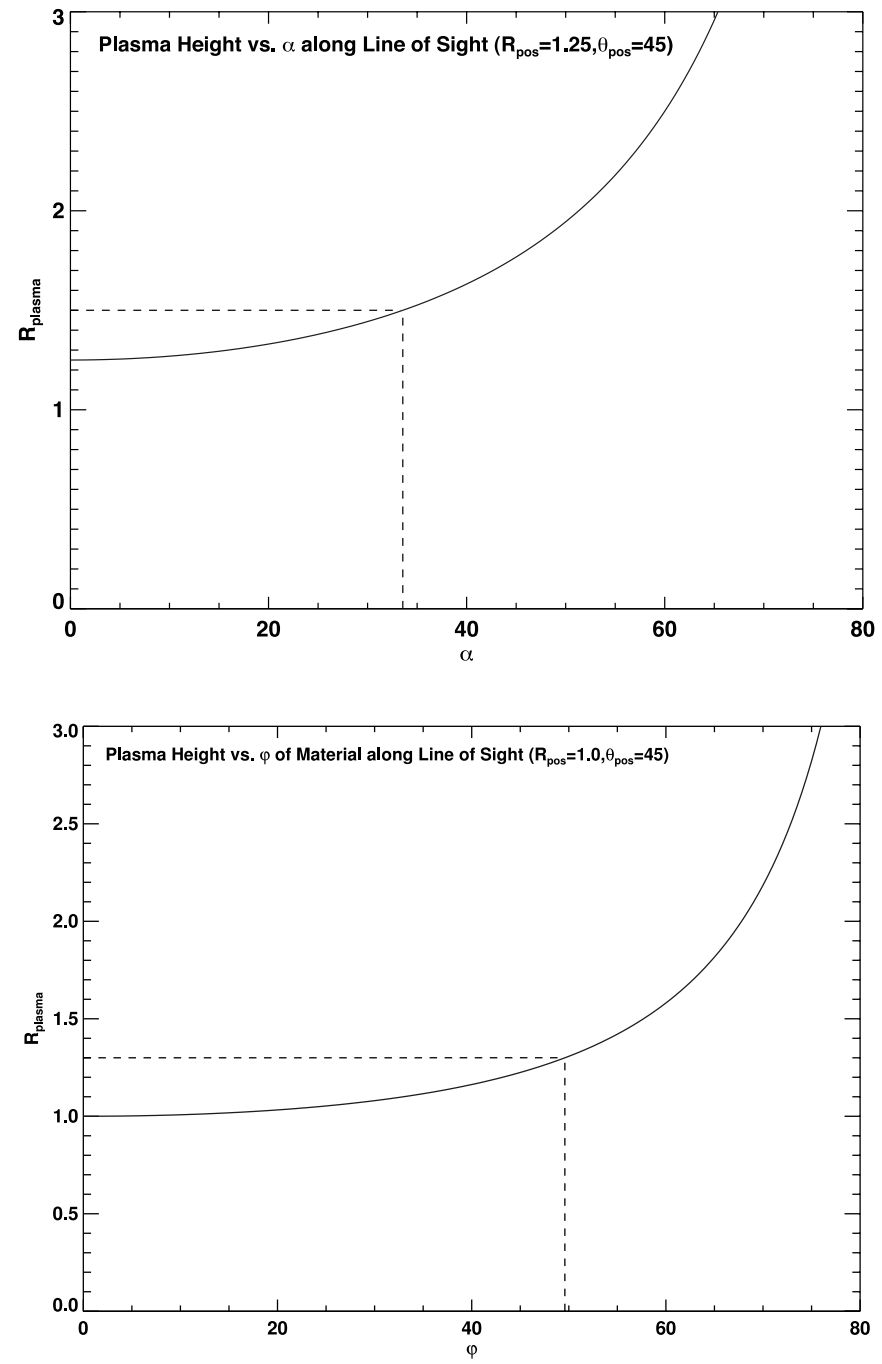

Fig. 7.-Top: Actual plasma height along the line of sight passing through $R_{\text {pos }}=1.25, \theta_{\text {pos }}=45^{\circ}$. The height $R_{\text {pos }}$ is given by the $y$-intercept of the curve. Bottom: Plasma height as a function of colongitude $\phi$ along a line of sight passing through $R_{\text {pos }}=1.0$ and $\theta_{\text {pos }}=45^{\circ}$. The dotted lines in both plots can be used to find the angles at which material at a given plasma height enters these lines of sight. [See the electronic edition of the Journal for a color version of this figure.]

always end up with exactly two values of $\alpha_{\text {rim }}$, one at a positive colongitude and one at a negative colongitude.

The most uncontaminated measurements of this axisymmetric cavity model will occur when our line of sight passes through more cavity material and less rim material, i.e., when $\alpha_{\text {cav }}$ is large. The top plot of Figure 8 displays a plot of $\alpha_{\text {cav }}$ versus $R_{\text {pos }}$ for $R_{\text {cav }}=0.25$ at various colatitudes. For simplification, the points of observation lie at the central colatitude of the cavity so that $\theta_{\mathrm{pos}}=\theta_{c}$. It is clear that, in general, higher values of $R_{\mathrm{pos}}$ within the cavity, i.e., lines of sight passing near the rim of the cavity, will yield less accurate measurements because they correspond to smaller values of $\alpha_{\text {cav }}$ and so have relatively large amounts of rim contributing to the intensity signal.

It is also instructive to consider the case in which our line of sight passes through the center of the cavity so that $R_{\text {pos }}=$ $1+R_{\text {cav }}$ and $\theta_{\text {pos }}=\theta_{c}$. In this case we see that we are in the cavity while $d_{x y}<R_{\text {cav }}$, or when $R_{\phi}-R_{\text {curve }}<R_{\text {cav }}$. Equation (6) simplifies to

$$
\alpha_{\mathrm{cav}_{c}}= \pm \arctan \sqrt{\left(\frac{R_{\mathrm{cav}}}{1+R_{\mathrm{cav}}}\right)^{2}+\frac{2 R_{\mathrm{cav}} \sin \theta_{c}}{1+R_{\mathrm{cav}}}} .
$$
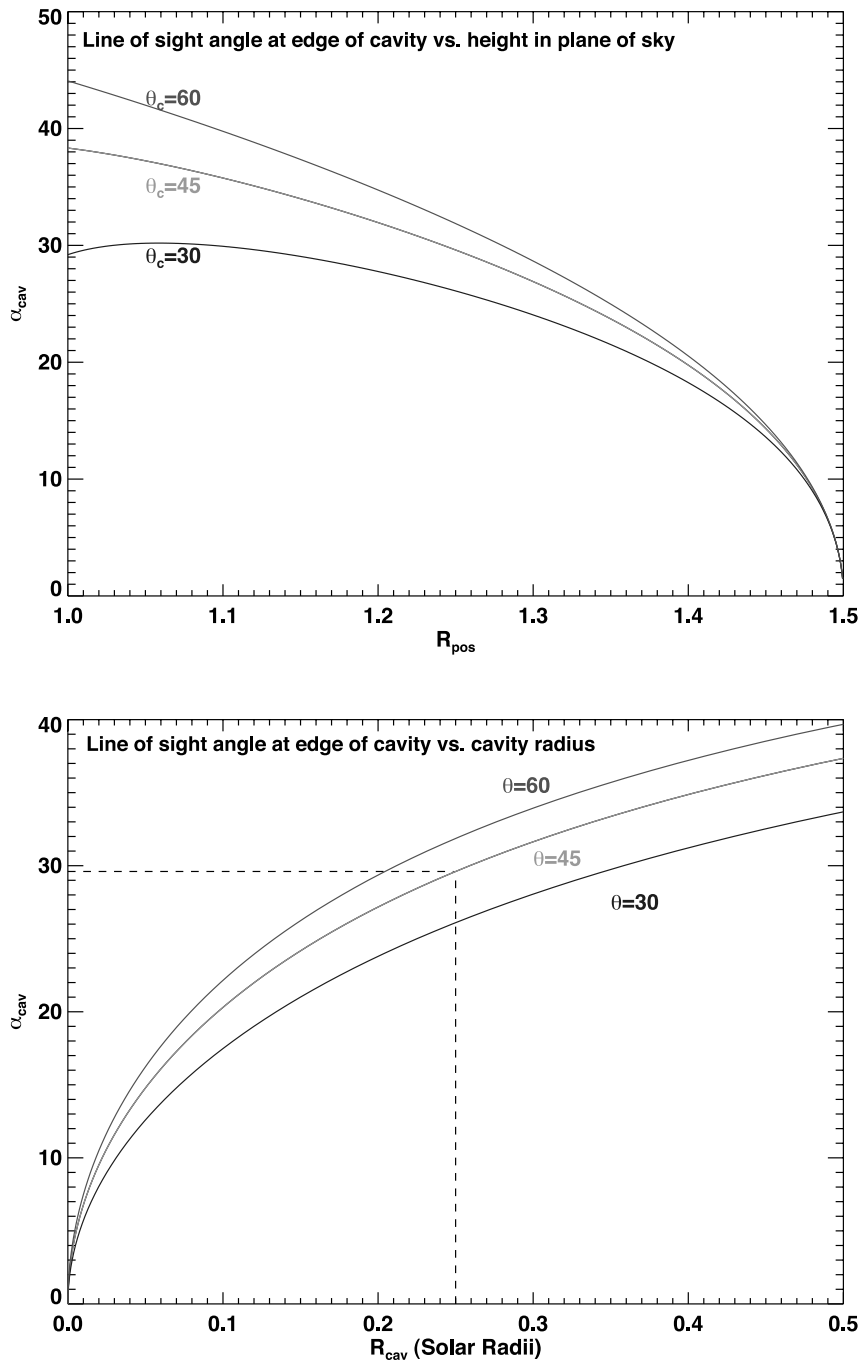

FIG. 8.-Top: Line-of-sight angle $\alpha_{\text {cav }}$ at the edge of the cavity as a function of $R_{\mathrm{pos}}$ for $R_{\mathrm{cav}}=0.25$ at various colatitudes $\left(\theta_{\mathrm{pos}}\right.$ has been assumed to be equal to $\left.\theta_{c}\right)$. Note that lines of sight nearest the photosphere will generally yield the best measurements in the absence of obstructing features near the photosphere. Bottom: Line-of-sight angle $\alpha_{\mathrm{cav}_{c}}$ at the edge of the cavity as a function of cavity radius for various colatitudes. Here $\alpha_{\mathrm{cav}_{c}}$ is calculated assuming that the line of sight passes through the center of the cavity at $R_{\mathrm{pos}}=1+R_{\mathrm{cav}}$ and $\theta_{\mathrm{pos}}=\theta_{c}$. The dotted lines show how the curves can be used to find the line-of-sight angle where the cavity rim projects onto a cavity of a given radius. [See the electronic edition of the Journal for a color version of this figure.]

The bottom plot of Figure 8 displays $\alpha_{\mathrm{cav}_{c}}$ as a function of $R_{\text {cav }}$ for varying colatitudes. Clearly, cavities with large radii and cavities near the equator have the largest values of $\alpha_{\mathrm{cav}_{c}}$.

Although equation (7) assumes a line of sight through the center of the cavity, all lines of sight along the central colatitude that lie below the cavity center will also pass through only cavity material for at least $\pm \alpha_{\mathrm{cav}_{c}}$, so long as

$$
\theta_{c}>\arcsin \left[\frac{\left(\sqrt{1+R_{\mathrm{cav}}}\right) / 2}{1+R_{\mathrm{cav}}}\right] .
$$

This limiting angle arises because the distance in the $x y$-plane between a point along the $\theta=\theta_{c}$ radial profile and the portion of the rim that projects onto it may increase with height at first for cavities at high enough latitudes (see Fig. 5, and bear in mind that the point and its corresponding projecting rim lie along a line parallel to the equator). If so, $\alpha_{\mathrm{cav}}$ will likewise increase (see, for 
example, the $\theta_{c}=30$ curve of the top plot of Fig. 8). For $0<$ $R_{\text {cav }}<1$, this limiting angle will have values between $30^{\circ}$ and $45^{\circ}$. For cavities with $\theta=\theta_{c}$ equatorward of this, however, $\alpha_{\text {cav }}$ will monotonically decrease with increasing $R_{\text {pos }}$.

We are now in a position to consider in general the types of cavities that are to be preferred for unobstructed viewing, in the context of the axisymmetric model. Obviously, a bigger cavity will have a rim that enters into the line of sight at a large angle $\alpha_{\text {cav }}$ and is to be preferred to a smaller cavity. It also is intuitive that a cavity that has less curvature, i.e., a larger radius of curvature, will provide better observations because our line of sight will be able to pass through more cavity. Since the radius of curvature is largest near the equator for our axisymmetric model, cavities with a colatitude $\theta_{c}$ closer to $90^{\circ}$ will work best. A brief aside here: the "ideal" viewing angle is for a cavity whose local orientation at the limb is along the line of sight, such that an underlying filament channel is oriented along a constant polar angle of the plane-of-sky projected solar disk (e.g., like slices on a pie; Wiik et al. 1994). In essence, cavities at high latitudes oriented in this way will have the same curvature as a same-sized cavity at and oriented along the equator. The optimal viewing would be short lived, however, since the plane of rotation for such a nonequatorial cavity is not aligned with that of solar rotation. Thus, the central colatitude $\theta_{c}$ would change with time, and only when it was most comparable to the angle of the filament channel with respect to the equator (on the projected solar disk) would the viewing be optimal. For the polar crown filament cavity that we will consider in this paper, the axisymmetric model is more appropriate, and, as we will demonstrate, because of its size, central colatitude and longitudinal extent, its departure from line-of-sight alignment is not significant.

\subsection{Departures from Axisymmetry}

Unfortunately, most cavities do not encircle the entire Sun. However, in order for a cavity to fit the parameters of our model, it does not need to be a complete torus, but only needs to be a torus for as long as our line of sight passes through it (see Fig. 9). In other words, the cavity needs to be torus shaped from the longitude at which the line of sight enters the cavity to the longitude at which the line of sight exits the cavity. If this holds true for all heights along a $\theta_{\text {pos }}$ profile, we call it effectively axisymmetric.

In general, we need to calculate, for all points $R_{\text {pos }}, \theta_{\text {pos }}$ under consideration, a minimum longitudinal extent $\phi_{\text {sym }}$, which the cavity must have on either side of the plane of the sky in order for it to have complete effective axisymmetry. We can get an idea about how axisymmetric a cavity is, however, by considering the $\theta_{\text {pos }}=\theta_{c}$ profile. Figure 9 shows a nonaxisymmetric torus as viewed from the north pole, in particular in the $x y$-plane corresponding to where the cavity touches the photosphere (for an equator-centered cavity, this will be identical to the plane of rotation; for all other colatitudes it will lie equatorward of the plane of rotation; see Fig. 5). The line of sight shown intersects the plane of the sky at the photosphere, and we see that at least for the earthward/bottom portion of the line of sight, the longitudinal extent of the cavity is large enough that the "cavity legs," i.e., the cavity rim where it bends down to the photosphere, have been occulted by the Sun's own disk (the line of sight only passes through the amount of rim material that it would for the axisymmetric case). The most stringent case for axisymmetry (that is, the largest $\phi$ ) occurs in this way for $R_{\text {pos }}=1$ (again subject to the constraint of eq. [8]). We can solve for this angle $\phi_{\mathrm{sym}_{c}}$ beyond which plasma lying at physical heights $r<R_{\text {cav }}$ projects below $R_{\text {pos }}=1$ (that is, is occulted by the Sun's disk). Setting

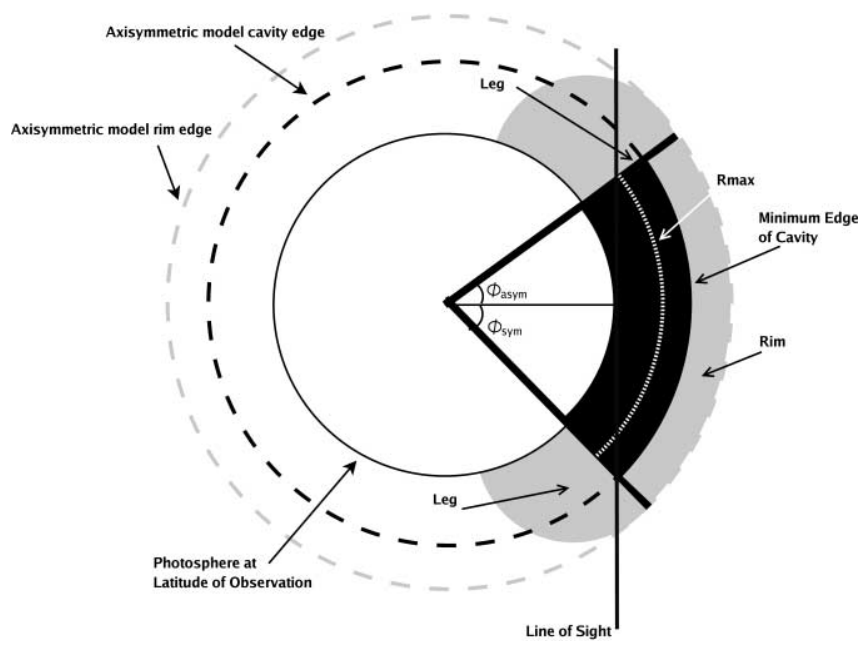

FIG. 9.-Diagram of a cavity that does not completely encircle the Sun, as viewed from the Sun's north pole. The $x y$-plane shown includes the point where the cavity touches the photosphere and is generally equatorward of the plane of rotation of the cavity. The gray region indicates rim and legs and the black region indicates cavity as in Fig. 4, but differs from that figure in that outside of the wedge of longitudes where the cavity is known to be, the "worst-case scenario" of contamination by bright rim is assumed. Dashed lines indicate the rim and cavity edge for the axisymmetric model. The dotted white line indicates a constant height $R_{\max }$ that is below the top of the cavity. The line of sight shown intersects the plane of the sky at $R_{\text {pos }}=1$. The cavity as shown here would have the required longitudinal extent $\left(\phi_{\text {sym }}\right)$ to be effectively axisymmetric on the earthward side but not on the other side, where the line of sight intersects cavity legs at heights that would be cavity in the axisymmetric model. Note however that the cavity is effectively axisymmetric on both sides if plasma lying above $R_{\max }$ does not contribute significantly.

$d=R_{\text {cav }}, \theta_{\text {pos }}=\theta_{c}$, and $R_{\text {pos }}=1$, we see equations (1) and (6) reduce to

$$
\phi_{\mathrm{sym}_{c}}= \pm \arctan \left[2 \sqrt{\left(R_{\mathrm{cav}}\right)^{2}+R_{\mathrm{cav}}}\right] .
$$

Due to the Sun's rotation, this longitudinal extent can be translated into a time period over which the cavity must maintain constant size and latitude. The corona rotates at about $13.5^{\circ}$ day $^{-1}$ as seen from the Earth, so the total number of days a cavity must maintain constant size and latitude for it to be axisymmetric on the middle day is $N_{\text {days }}=2 \phi_{\text {sym }_{c}} / 13.5$. Figure 10 displays a graph of $N_{\text {days }}$ versus $R_{\text {cav }}$ for $R_{\text {pos }}=1$ and $\theta_{\text {pos }}=\theta_{c}$. We can see that it is necessary for a cavity to exist for several days in order for it to be axisymmetric on the middle day and that large cavities require more days for axisymmetry.

If a cavity fails this most stringent axisymmetry condition, there are two other considerations that can be taken into account. First of all, the cavity may be effectively axisymmetric for planeof-sky heights above the photosphere. A line of sight shifted far enough to the right of the photosphere-grazing one shown in Figure 9 will not intersect the cavity legs. Given a known longitudinal extent $\phi_{\text {obs }}$ along with $R_{\text {cav }}, \theta_{c}$, and setting $d=R_{\text {cav }}$,

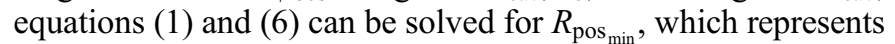
the minimum plane-of-sky height above which the cavity is effectively axisymmetric along the central colatitude. Second, if the cavity is large enough that density has fallen to subnoise levels before reaching the cavity rim, the criterion for axisymmetry is somewhat less stringent. This is demonstrated with the dotted line in Figure 9-if plasma above $R_{\max }$ is essentially unobservable, the cavity as shown in that figure would be effectively axisymmetric on both sides. In such cases, using equations (1) and (2) 


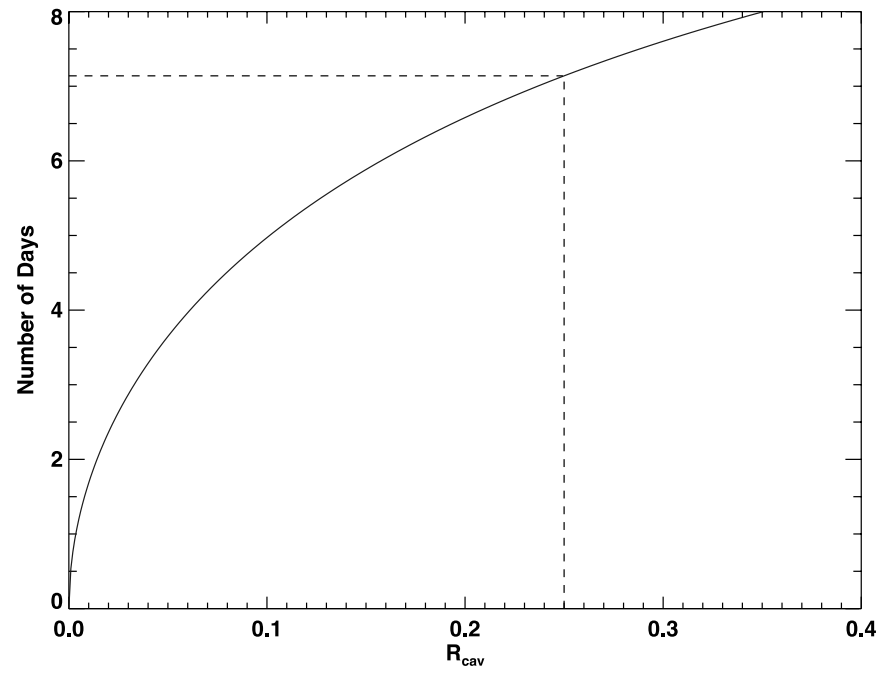

FIG. 10.- Total number of days that the cavity must maintain constant size and latitude to be axisymmetric on the middle day as a function of $R_{\text {cav }}$. The plot assumes $R_{\mathrm{pos}}=1$ and $\theta_{\mathrm{pos}}=\theta_{c}$. [See the electronic edition of the Journal for a color version of this figure.]

and setting $R_{\text {plasma }}=R_{\max }$, we see that the criterion for axisymmetry (along a central colatitude, $\theta_{\text {pos }}=\theta_{c}$ ) becomes

$$
\phi_{\mathrm{sym}_{\max }}= \pm \arctan \left(\sqrt{\frac{R_{\mathrm{max}}^{2}-R_{\mathrm{pos}}^{2}}{R_{\mathrm{pos}}^{2} \sin ^{2} \theta_{c}}}\right) .
$$

We can then set $R_{\text {pos }}=1$ and solve for the condition of complete effective axisymmetry or, given a $\phi_{\text {obs }}$, invert equation (10) and solve for a $R_{\text {pos }_{\text {min }}}$ as above.

Finally, to the extent that effective axisymmetry does not exist in an observed cavity, the contribution to the line-of-sight integral from the cavity legs may be explicitly included as a measurement uncertainty. As we will show below, since white-light $p B$ observations are dominated by contributions from material in the plane of the sky, such uncertainties may be quite small.

\subsection{Impact of Cavity Rim versus Cavity Legs}

To summarize, there are two main contributors to contaminating a pure cavity signal (ignoring for the moment substructure within the cavity), the cavity rim and the cavity legs. The larger the height at which an observed plane-of-sky point $\mathrm{O}$ is, the more contamination it will generally receive from the cavity rim. On the other hand, the condition for effective axisymmetry where the legs do not project into the line of sight is satisfied for less longitudinal cavity extent with increasing plane-of-sky height (see Fig. 9). A bigger cavity is to be preferred to avoid rim projection, as it means less curvature and a higher top. However, to satisfy axisymmetry, a smaller cavity is to be preferred, as it requires less longitudinal extent (see eq. [9]). Thus, there is a tradeoff, and in choosing a cavity for its lack of obstruction, both legs and rims need to be considered.

\section{WHITE-LIGHT CAVITY}

\subsection{Cavity Properties}

In this section of the paper we will describe the cavity that was observed in white light by the MLSO/MK4 coronagraph from 2006 January 25 to 30 (Fig. 2). We have chosen this cavity because it is large, possesses high contrast to its surrounding helmet
TABLE 1

Cavity Properties

\begin{tabular}{ccccc}
\hline \hline Date & $R_{\text {cav }}$ & $\theta_{c}$ & $\alpha_{\mathrm{cav}_{c}}$ & $\phi_{\mathrm{sym}_{c}}$ \\
\hline 2006 Jan 25.... & $0.230 \pm 0.030$ & $124.80 \pm 0.74$ & $30.32_{-1.65}^{+1.48}$ & $46.77_{-2.35}^{+2.09}$ \\
2006 Jan 27..... & $0.260 \pm 0.035$ & $128.44 \pm 0.63$ & $31.17_{-1.71}^{+1.52}$ & $48.86_{-2.46}^{+2.17}$ \\
2006 Jan 28..... & $0.270 \pm 0.045$ & $129.64 \pm 0.67$ & $31.40_{-2.14}^{+1.85}$ & $49.51_{-3.10}^{+2.65}$ \\
2006 Jan 29..... & $0.285 \pm 0.045$ & $132.40 \pm 0.89$ & $31.54_{-2.07}^{+1.81}$ & $50.44_{-2.94}^{+2.52}$ \\
2006 Jan 30..... & $0.260 \pm 0.030$ & $131.36 \pm 0.54$ & $30.69_{-1.45}^{+1.32}$ & $48.86_{-2.09}^{+1.87}$ \\
\hline
\end{tabular}

Notes.-Cavity properties for each date of observation. The values of $\alpha_{\text {cav }_{c}}$ indicate the scattering angle where the edge of the cavity would enter the line of sight intersecting the plane-of-sky cavity center $\left(R_{\text {pos }}=1+R_{\text {cav }}\right)$ for the axisymmetric model (eq. [7]). The values of $\phi_{\mathrm{sym}_{c}}$ indicate the colongitude of the edge of the cavity along the line of sight intersecting the coronal base $\left(R_{\mathrm{pos}}=1\right)$ at the cavity central colatitude for the axisymmetric model (eq. [9]).

streamer, and is relatively close to the equator. See Table 1 for a list of its cavity properties. Furthermore, the cavity exhibits little change in size or shape for at least 6 days and thus encircles at least $81^{\circ}$ of the Sun. Unfortunately, there are no data for January 24 so we cannot be sure whether the cavity was visible on this day, and although it is noticeable on the $31 \mathrm{st}$, it is much less distinct than on previous days. To be conservative, we therefore only consider the cavity between January 25 and 30. Table 2 shows the number of days on either side of an observation that would ensure complete effective axisymmetry for the central colatitude (calculated according to eq. [9]) and the number of days that the cavity is actually observed on either side of each observation along with the longitudinal extent thus implied $\left(\phi_{\text {obs }}\right)$. It is clear that the cavity comes closest to being axisymmetric on January 27 and 28. Bear in mind that the number of days required is calculated for $R_{\text {pos }}=1$ and that the number of days required is less at higher altitudes (eq. [8] is satisfied for all the days for this cavity). The final column of Table 2 shows the minimum height above which the cavity is axisymmetric $R_{\text {pos }_{\text {min }}}$ at the colatitude $\theta_{c}$, based on the observed longitudinal extent $\phi_{\text {obs }}$.

\subsection{Nature of Cavity Plasma}

The white-light observable $p B$ measures photospheric light scattered off of coronal electrons and is defined by

$$
p B\left(R_{\mathrm{pos}}, \theta_{\mathrm{pos}}\right)=\int N_{e}(r, \theta, \phi) C(r) d \alpha .
$$

Here $N_{e}$ is the electron density and $C(r)$ is a Thomson scattering function that depends on radial height (Billings 1966). In our model for coronal cavities, it is inevitable that any chosen line of sight will pass through at least the rim of the cavity, if not its legs. It may seem as though any measurement of $p B$ will inescapably be subject to contamination from this noncavity material. However, with regards to the cavity rim, we must remember that the images of cavities such as those in Figures 1 and 2 have been multiplied by a radial gradient function that enhances the comparative brightness of structures at greater heights. In fact, the cavity top is not clearly evident in images where the radial gradient function has not been applied. The density of the corona falls off very rapidly with height, so features such as the cavity top are actually dimmer than features below them due to their lower density of scattering electrons (see Fig. 3, bottom). Furthermore, features at higher altitudes are farther away from the Sun and thus see a smaller intensity of photospheric light to scatter. The cavity legs, on the other hand, do by definition curve down to the photosphere and so are filled with higher density plasma, 
TABLE 2

CAVITY AXISYMmetry

\begin{tabular}{|c|c|c|c|c|}
\hline Date & Days Required & Days Observed & Longitudinal Extent $\phi_{\text {obs }}$ & $R_{\text {pos }_{\min }}$ \\
\hline 2006 Jan $25 \ldots \ldots \ldots \ldots . .$. & $3.46_{-0.17}^{+0.16}$ & 0.5 before, 5.5 after & 6.75 before, 74.25 after & $1.45,1.0$ \\
\hline 2006 Jan $27 \ldots \ldots \ldots \ldots . . .$. & $3.62_{-0.18}^{+0.16}$ & 2.5 before, 3.5 after & 33.75 before, 47.25 after & $1.32,1.05$ \\
\hline 2006 Jan $28 \ldots \ldots \ldots \ldots \ldots$ & $3.67_{-0.23}^{+0.18}$ & 3.5 before, 2.5 after & 47.25 before, 33.75 after & $1.07,1.34$ \\
\hline 2006 Jan $29 \ldots \ldots \ldots \ldots . . .$. & $3.74_{-0.22}^{+0.19}$ & 4.5 before, 1.5 after & 60.75 before, 20.25 after & $1.0,1.51$ \\
\hline 2006 Jan $30 \ldots \ldots \ldots \ldots . . . . .$. & $3.62_{-0.16}^{+0.14}$ & 5.5 before, 0.5 after & 74.25 before, 6.75 after & $1.0,1.52$ \\
\hline
\end{tabular}

Notes.-This table lists the number of days the cavity must maintain constant latitude and size before and after an observation for it to have complete effective axisymmetry (see text). The table also lists a conservative estimate of the number of days before and after each date that we observe the cavity to be visible (assuming that observations occur midday the day of observation), and the longitudinal extent that this implies $\left(\phi_{\mathrm{obs}}\right)$. The last column indicates the lowest line-of-sight height $R_{\text {pos }}$ at $\theta_{\text {pos }}=\theta_{c}$ for which the cavity is effectively axisymmetric for the central colatitude, using the values of $\phi_{\text {obs. }}$. The two values correspond to non-Earthward and Earthward sides, respectively.

but since our observed cavity is nearly axisymmetric, the legs only enter into the line of sight at relatively large values of lineof-sight angle $\alpha$, if at all.

The fact is, most of the scattered light contributing to a $p B$ signal is scattered between angles corresponding to low values of $\alpha$, or equivalently, the $p B$ signal is dominated by contributions from plasma near the plane of the sky (Hundhausen 1993). Figure 11 displays a plot of $p B$ versus the scattering angle $\alpha$, assuming a density falloff of $R^{7}$ and a spherical body (non-point source) of $1 R_{\odot}$, with limb darkening taken into account. Any $p B$ measurement corresponds to the integral along the line of sight (the area under the curve), but we can see that the signal is dominated by material at small scattering angles, even for relatively low heights.

We can illustrate this more precisely for the white-light cavity that we are considering in this paper, assuming for the moment that it is axisymmetric. According to our model, the cavity rim enters the cavity center line of sight at scattering angles of just over $30^{\circ}$. A line of sight through the center of our cavity has an altitude of roughly $R_{\mathrm{pos}}=1.25$ (corresponding to the middle curve in Fig. 11), and we can calculate the fraction of the area under the curve that lies between the dashed lines in the figure to determine the fraction of light that originates from angles be-

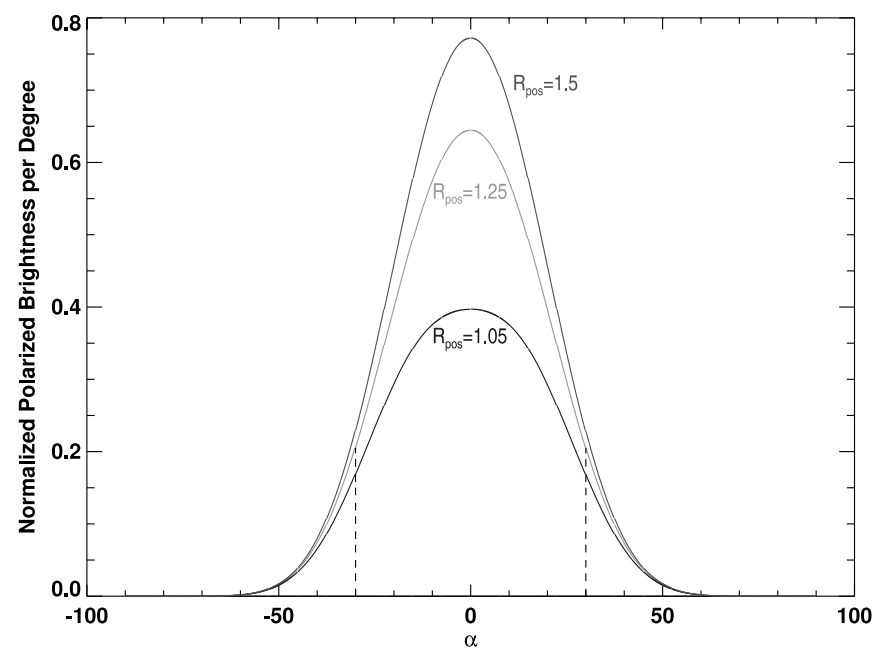

Fig. 11.-Plot of $p B$ as a function of scattering angle for various altitudes of the line of sight. Limb darkening is taken into account with $\mu=0.585$. The curves have been normalized to demonstrate how brightness drops off faster with $\alpha$ at higher altitudes. Without the normalization, lower values of $R_{\text {pos }}$ would have higher peak brightnesses. The curves assume a spherically symmetric density that falls off as $R^{7}$. The dashed lines indicate the values of $\alpha= \pm 30^{\circ}$ (after Hundhausen 1993). [See the electronic edition of the Journal for a color version of this figure.] tween $\pm 30^{\circ}$. It turns out that at this height $88.69 \%$ of the light comes from within these angles for an axisymmetric model. Even if we adjust the curve in Figure 11 so that the density in the cavity legs is double the density at the same height inside of the cavity, we find that $79.66 \%$ of the light emanates from within the cavity. For our cavity, it is clear that scattered light from within the cavity dominates the signal of $p B$ if we look along a line of sight that cuts through the middle or bottom portion of the cavity. Although the cavity rim does contribute to the signal, it cannot account for all of the light scattered along this line of sight. If the cavity were completely empty and scattered no light, the cavity should appear only $11.31 \%$ as bright as the rim for the same value of $R_{\text {pos }}$ (see also Fisher \& Munro [1984] for similar analysis of the cavity behind a coronal mass ejection). However, Figure 3 (top left) displays a plot of $p B$ as a function of latitude at $R_{\text {pos }}=1.2$ for our cavity on January 27 . Note that even the dimmest part of the cavity is much more than one-eighth as bright as the brightest parts of the cavity rim. Hence, the cavity rim cannot account for all the light so light is being scattered from within the cavity. Clearly, the cavity is not empty. In $\S 4$ we will calculate the cavity's actual density and describe how the contributions of rim and legs can explicitly be used as correction factors and uncertainties on the measured cavity $p B$.

\section{CAVITY DENSITY CALCULATION}

To calculate the density of our cavity we use a Van de Hulst inversion of the measurements of $p B$ to obtain a radial density profile (Van de Hulst 1950). It should be noted that a Van de Hulst inversion assumes cylindrical symmetry along the line of sight. In other words, it assumes that the radial density falloff is the same at all scattering angles along the line of sight. As long as the line of sight is within the cavity, a Van de Hulst inversion is appropriate because the same radial density falloff is assumed at all points within the cavity. However, once we reach the scattering angle of $\alpha_{\text {cav }}$ calculated in Table 1, we exit the cavity into the cavity rim, where the density falloff is different and the cylindrical symmetry is broken. Eventually, our line of sight passes out of the helmet streamer into the surrounding material, breaking the symmetry once again. Moreover, to the extent that the cavity is not axisymmetric, the line of sight can intersect the cavity legs (Fig. 9), again breaking the assumed cylindrical symmetry.

\subsection{Cavity Measurement Uncertainties}

To deal with this problem, we can add error bars to the measured brightness for each point that we measure. For the moment, let us assume that the cavity is axisymmetric. We can decompose 
the measured value of $p B$ into the brightness emanating from cavity material, rim material, and noncavity material so that

$$
p B_{\text {meas }}=\left.2 p B_{\text {cav }}\right|_{0} ^{\alpha_{\text {cav }}}+\left.2 p B_{\text {rim }}\right|_{\alpha_{\text {cav }}} ^{\alpha_{\text {rim }}}+\left.2 p B_{\text {non }}\right|_{\alpha_{\text {rim }}} ^{90} .
$$

Here the $p B_{\text {cav }}$ refers to contributions from material in the cavity, i.e., contributions by material with the density of cavity material. The $p B_{\text {rim }}$ term refers to material with a density profile of a helmet streamer, while the $p B_{\text {non }}$ term refers to whatever type of material surrounds the helmet streamer. We determine $\alpha_{\text {rim }}$ using equation (6) and setting $d=R_{\text {rim }}=R_{\text {cav }}+0.2$. For a given value of $R_{\text {pos }}$ and a given range in scattering angles, we can calculate $p B_{\text {rim }}$ and $p B_{\text {non }}$ terms if we know the density profiles of the contributing material. In this paper, we use the density profiles for helmet streamers and coronal holes as calculated by Gibson et al. (1999) and Guhathakurta et al. (1999), respectively. The Van de Hulst inversion assumes cylindrical symmetry, so in order for it to work properly we must give it the value of $p B$ that we would measure if all of the material along the line of sight had the same density profile as the cavity material. So the inversion requires the value

$$
\left.p B_{\mathrm{cav}}\right|_{-90} ^{90}=\left.2 p B_{\mathrm{cav}}\right|_{0} ^{\alpha_{\mathrm{cav}}}+\left.2 p B_{\mathrm{cav}}\right|_{\alpha_{\mathrm{cav}}} ^{\alpha_{\mathrm{rim}}}+\left.2 p B_{\mathrm{cav}}\right|_{\alpha_{\mathrm{rim}}} ^{90} .
$$

Combining this equation with the previous equation, we find that the value for $p B$ that we should input into the inversion is

$$
\begin{aligned}
\left.p B_{\mathrm{cav}}\right|_{-90} ^{90}= & p B_{\text {meas }}+\left(\left.2 p B_{\mathrm{cav}}\right|_{\alpha_{\mathrm{cav}}} ^{\alpha_{\mathrm{rim}}}-\left.2 p B_{\mathrm{rim}}\right|_{\alpha_{\mathrm{cav}}} ^{\alpha_{\mathrm{rim}}}\right) \\
& +\left(\left.2 p B_{\mathrm{cav}}\right|_{\alpha_{\mathrm{rim}}} ^{90}-\left.2 p B_{\mathrm{non}}\right|_{\alpha_{\mathrm{rim}}} ^{90}\right)
\end{aligned}
$$

The terms inside the parentheses represent the correction factors for our measurements. If material with a density higher than that of the cavity lies along the line of sight (i.e., the cavity rim), it will contribute more brightness than cavity material would have contributed, so we add a negative correction. If material with a density lower than that of the cavity lies along the line of sight (i.e., a coronal hole), we may have to add a positive correction. Since we do not know the cavity density, we choose extreme values corresponding to that for a coronal streamer and that for a coronal hole and obtain a maximum uncertainty in either direction. We also must determine the type of material that surrounds the helmet streamer and contributes to the $p B_{\text {non }}$ term. Helmet streamers are often surrounded by coronal holes, but if we can see from observations that an adjacent helmet streamer projects into the cavity's line of sight at high scattering angles, we can take this into account. We thus determine upper and lower limits on the correction factor corresponding to the extreme choices for cavity material (hole vs. streamer). This correction factor is generally negative, as it primarily accounts for the projection of the rim onto the cavity. We also add an instrumental uncertainty of $7.5 \%$ to $p B_{\text {meas }}$, appropriate for January 27 , which had clear skies and a recently cleaned objective lens (Elmore et al. 2003). Thus, we can calculate the corrected $p B_{\text {cav }}$, center it between positive and negative uncertainties, and input this value into the Van de Hulst calculations.

The cavity that we are fitting does not quite have the required longitudinal extent to meet the most stringent requirements for axisymetry, as can be seen in Table 2 . To deal with this problem, we may visualize the cavity as a partial torus that has been sliced at a certain longitude in front of and behind the cavity, as shown in Figure 9. To determine these cavity leg longitudes, we observe the number of days before and after the date of observation that the cavity is clearly visible. Using these longitudes, we calculate two new values of $\alpha_{\text {cav }}$ at which the line of sight exits the cavity. Then, if these two values of $\alpha_{\text {cav }}$ are smaller than the $\alpha_{\text {cav }}$ calculated assuming axisymmetry, we use them in our uncertainty calculations because they will create larger uncertainties. We assume that the cavity legs extend a distance $0.5 R_{\odot}$ along the line of sight from $\alpha_{\text {cav }}$, and so are able to likewise calculate an adjusted value of $\alpha_{\text {rim }}$.

\subsection{Rim Measurement Uncertainties}

The process for calculating uncertainties in measurements of $p B$ for the cavity rim is quite similar. Once again, the inversion requires cylindrical symmetry along the line of sight, so we must adjust our measurements in the same manner described above. As discussed above, a line of sight at low altitude through the (planeof-sky) poleward cavity rim may pass in and out of the cavity, yielding a total of four values for $\alpha_{\text {cav }}$ (two on either side of the plane of the sky). So, assuming that the cavity and rim are axisymmetric, the measured value of $p B$ for the rim is

$$
\begin{aligned}
p B_{\text {meas }}= & \left.2 p B_{\text {rim }}\right|_{0} ^{\alpha_{\mathrm{cav}_{1}}}+\left.2 p B_{\mathrm{cav}}\right|_{\alpha_{c a v 1}} ^{\alpha_{\mathrm{cav}_{2}}} \\
& +\left.2 p B_{\text {rim }}\right|_{\alpha_{\mathrm{cav}_{2}}} ^{\alpha_{\mathrm{rim}}}+\left.2 p B_{\mathrm{non}}\right|_{\alpha_{\mathrm{rim}}} ^{90} .
\end{aligned}
$$

We calculate the values of $\alpha_{\mathrm{cav}_{1}}$ and $\alpha_{\mathrm{cav}_{2}}$ using equation (6) and setting $d$ equal to $R_{\text {cav }}$, and we calculate $\alpha_{\text {rim }}$ by setting $d$ equal to $R_{\text {rim }}$. In the case where the line of sight does not pass through the cavity, we can ignore the $\alpha_{\text {cav }}$ terms. We treat asymmetry and then add the most conservative error bars to the measurements using the same method described above.

\subsection{Inverting the Measurements}

In this paper, we apply the Van de Hulst inversion to the data for January 27. As a comparison of values of $\phi_{\mathrm{Sym}_{c}}$ and $\phi_{\mathrm{obs}}$ in Tables 1 and 2 indicates, this day very nearly meets the criteria for axisymmetry on the Earthward side but falls short on the non-Earthward side. We therefore explicitly include contributions from bright cavity legs in our error analysis as discussed above. Although January 28 is axisymmetric to an equal extent, the presence of an active region (see Fig. 12, left) on the equatorward side of the cavity makes accurate measurements more difficult. While this active region does not affect the cavity on January 25 , the required longitudinal extent for axisymmetry is lacking on this day. For January 27, we perform an inversion on both the cavity and the cavity rim in order to obtain a meaningful density ratio. To perform the inversion, we first choose the set of colatitudes corresponding to an unobstructed location in the cavity and another corresponding to the peak brightness of the cavity rim, as shown in Figure 12 (left, vertical black line segments). Unfortunately, the active region that complicates matters on January 28 is also present to a lesser degree on the 27 th. Consequently, we perform our measurement a few degrees to the poleward side of the center of the cavity. The cavity is centered at $\theta_{c}=232^{\circ}$, but we perform our cavity measurements at $\theta_{\text {pos }}=$ $228^{\circ}$ and our cavity rim measurements at $\theta_{\text {pos }}=219^{\circ}$.

We use $R_{\text {cav }}=0.225$, which is the lower limit on the error bars for the cavity radius of January 27 , and moreover is less than the minimum observed cavity radius on January 25 . We 

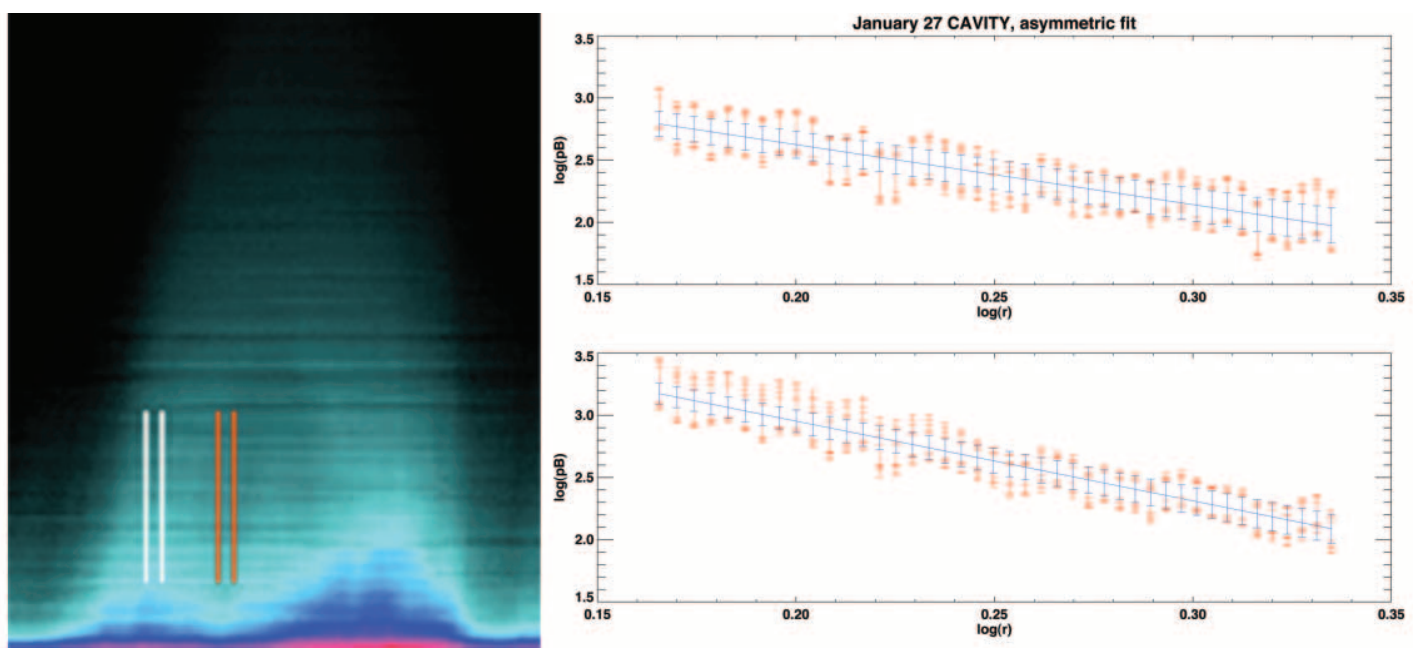

FIG. 12.-Left: MLSO/MK4 pB image of the cavity on January 27 in polar coordinates $(r$ vs. $\theta)$. Note the active region that interferes with measurements near the

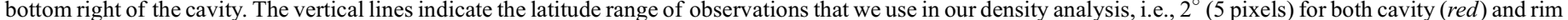

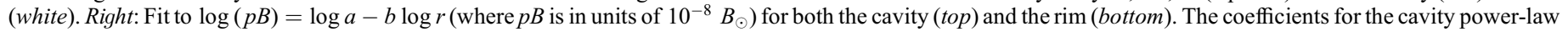

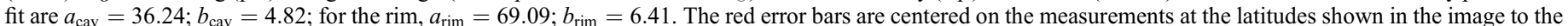

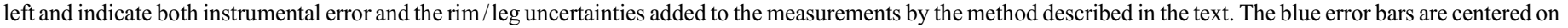
the line of best fit and indicate the uncertainty in the fit.

also limit the inversion to a height range of $1.18-1.4 R_{\odot}$. Below $1.18 R_{\odot}$, the cavity is contaminated by brightening from features near the photosphere. Since cavities observed in white light are large, they can contain magnetic substructures at lower altitudes that could interfere with measurements. Above about $1.4 R_{\odot}$, the cavity begins to merge with the surrounding helmet streamer and contamination from the cavity rim becomes significant. Consequently, we do not calculate density at these altitudes.

Figure 12 (right) displays log-log plots of radial profiles of $p B$ for the cavity and the rim. The red error bars are calculated as described above: note that roughly half (ranging from $40 \%$ to $60 \%$ depending on height) of the error is due to instrumental uncertainties, and the rest is due to the potential contributions from noncavity material. We fit a linear falloff with $\log (p B)=\log a-$ $b \log (r)$, which corresponds to a power-law falloff of the form $p B=a r^{-b}$. While a six-parameter power-law fit has been used for density calculations of helmet streamers by Gibson et al. (1999), the altitudes considered in that calculation ranged from very near the solar photosphere to several $R_{\odot}$. Since our measurement is restricted to a narrow range in altitude, a two-parameter fit is sufficient. After calculating the parameters $a$ and $b$, we perform a Van de Hulst inversion, which utilizes these coefficients to calculate the radial density profile.

\subsection{Results}

The results of the inversions are shown in Figures 13 and 14. Figure 13 compares the $p B$ and density profiles for the cavity and the cavity rim on January 27 . As expected, the cavity rim is clearly more dense than the cavity for the range of heights measured. The density depletion with respect to the cavity rim is defined as

$$
\frac{\Delta N}{N}=\frac{N_{\text {rim }}-N_{\text {cav }}}{N_{\text {rim }}} .
$$

The cavity has a $40 \%$ density depletion at lower altitudes but much less depletion at higher altitudes. This appears to be a result of the cavity's markedly slower density falloff. Although the cavity becomes contaminated by light from the cavity rim at higher altitudes, we have taken this into consideration in our error analysis. The flatness of the cavity density profile relative to that of the rim is clear at all heights measured. We will discuss the implications for the cavity temperature and magnetic structure in $\S 5$.

Figure 14 displays the calculated density for the cavity and cavity rim on January 27 , as well as the density for a coronal hole and bright helmet streamer as calculated during the Whole Sun Month (WSM; Gibson et al. 1999; Guhathakurta et al. 1999). The cavity rim has a similar density profile to the WSM helmet streamer. It is clear that the cavity has a significantly higher density than the WSM coronal hole, however. The coronal hole has a density depletion of about $50 \%$ with respect to the cavity at low altitudes and a depletion of at least $80 \%$ at higher altitudes. The cavity has a falloff in density that is more gradual than both the coronal hole and the coronal helmet streamer.

\section{TEMPERATURE AND MAGNETIC STRUCTURE OF CAVITIES}

In order to understand the cavity as a magnetohydrostatic equilibrium state, it is necessary to determine additional information about its thermal and magnetic structure. Let us first consider whether we can learn something about cavity temperature and magnetic field structure using only the radial profiles of density, as determined above, within the cavity versus the surrounding helmet streamer (cavity rim).

\subsection{Hydrostatic Temperature Analysis}

Because there are no magnetic forces along a magnetic field line, if one assumes that temperature is constant along the field line, it can be directly solved for given density (assuming that dynamic forces are negligible). Indeed, if the field line direction varies relative to the radial direction at an angle $\lambda,(d s=$ $d r / \cos [\lambda(s)])$, the gas pressure gradient along a field line is balanced by the component of gravity along that field line:

$$
\begin{gathered}
\frac{d P_{\mathrm{gas}}(s)}{d s}=-\frac{C_{\mathrm{gas}} N_{e}(s)}{r^{2}} \cos [\lambda(s)], \\
d P_{\mathrm{gas}_{\mathrm{f}}}(r)=-\frac{C_{\mathrm{gas}} N_{e_{\mathrm{f}}}(r)}{r^{2}} d r .
\end{gathered}
$$



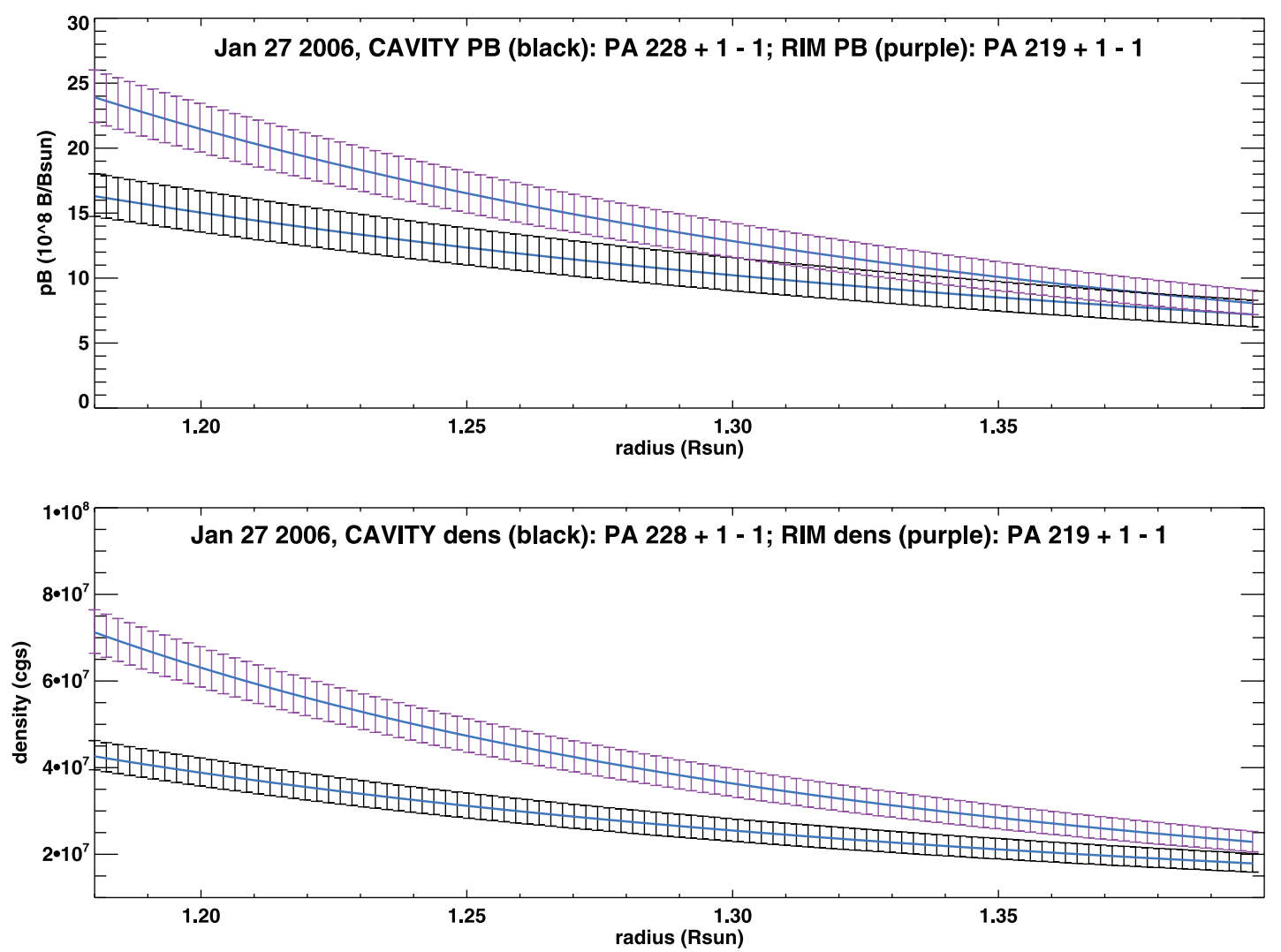

Fig. 13. - Plot of $p B(t o p)$ and density (bottom) profiles for the cavity (black) and the cavity rim ( purple) on January 27. The blue lines show the fits, with coefficients for the linear power-law $p B$ profile as given in Fig. 12 and coefficients for the hydrostatic density profile $\log N_{e}(r)=\beta+(\delta / r)($ see $\S 5.1)$ of $\beta_{\text {cav }}=12.00 ; \delta_{\text {cav }}=6.57$; $\beta_{\text {rim }}=10.79 ; \delta_{\text {rim }}=8.60$.

Here

$$
C_{\mathrm{gas}}=\frac{1+4 \alpha_{\mathrm{He}}}{1+2 \alpha_{\mathrm{He}}} G M_{\odot} m_{p}
$$

Here $P_{\text {gas }}$ is the gas pressure, $N_{e}$ is electron number density, and we will assume a standard photospheric helium abundance

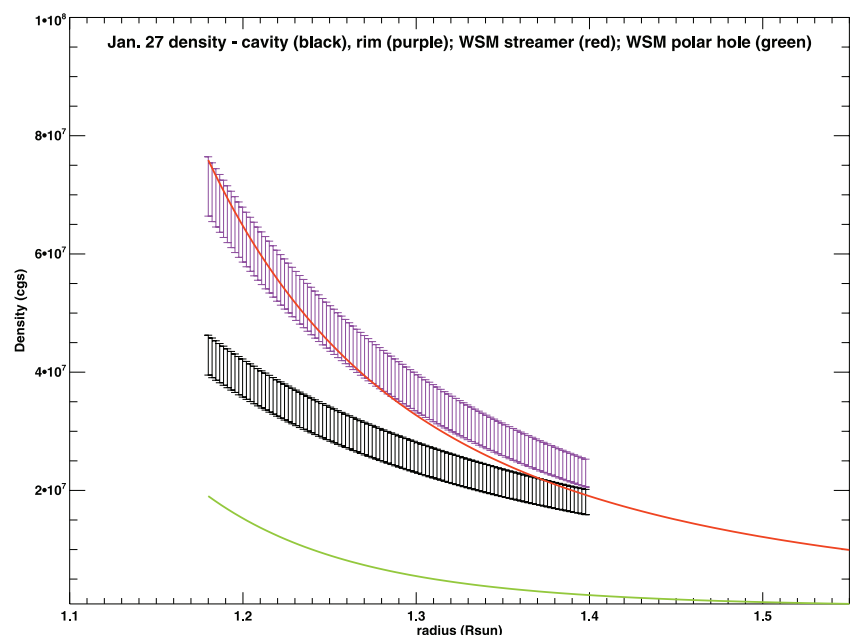

Fig. 14.- Results for the radial density profile of the cavity (black) and rim ( purple) on January 27 compared to density profiles for the WSM coronal hole (green; Guhathakurta et al. 1999) and the WSM helmet streamer (red; Gibson et al. 1999). (relative to hydrogen) of $\alpha_{\mathrm{He}}=0.1$ (Habbal et al. 1993). For an isothermal plasma, density and pressure are related by

$$
N_{e}=\frac{1+2 \alpha_{\mathrm{He}}}{2+3 \alpha_{\mathrm{He}}} \frac{P}{k T} .
$$

And so equation (18) can be solved for density, i.e.,

$$
\begin{gathered}
\frac{d N_{e_{\mathrm{f}}}(r)}{N_{e_{\mathrm{f}}}(r)}=-\frac{1}{h_{0}} \frac{d r}{r^{2}}, \\
h_{0}=\frac{k T}{C_{\mathrm{gas}}} \frac{2+3 \alpha_{\mathrm{He}}}{1+2 \alpha_{\mathrm{He}}}, \\
N_{e_{\mathrm{f}}}(r)=N_{e_{\mathrm{f}}}\left(r_{0}\right) \exp \left(\frac{1}{h_{0} r}-\frac{1}{h_{0} r_{0}}\right) .
\end{gathered}
$$

The variable $h_{0}$ is a hydrostatic scale height parameter for density and pressure: because we are in spherical geometry where gravity decreases with height, the effective $1 / e$ scale height increases with height as $h_{0} r_{0} r$ (Guhathakurta et al. 1992).

We use the subscript " $\mathrm{fl}$ " in the above equations to indicate that the integral of equation (18) must in general be performed independently for each field line. However, if a set of field lines possess a common temperature and a uniform density at their lower boundary, equation (18) can be integrated with respect to $r$ for a hydrostatic density and pressure distribution in that region that is independent offield line geometry (Priest 1982). We assume that the cavity and rim are two such regions, and fit the 


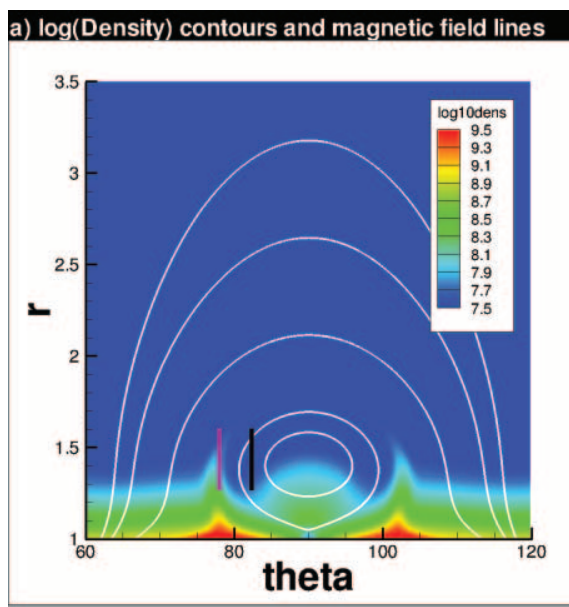

b) Field strength contours and field lines

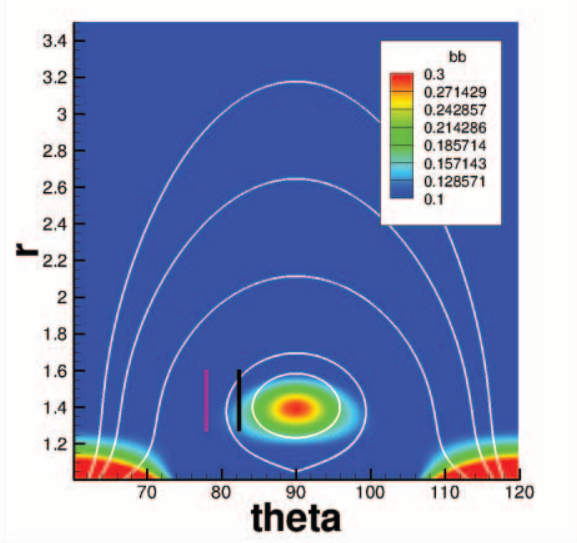

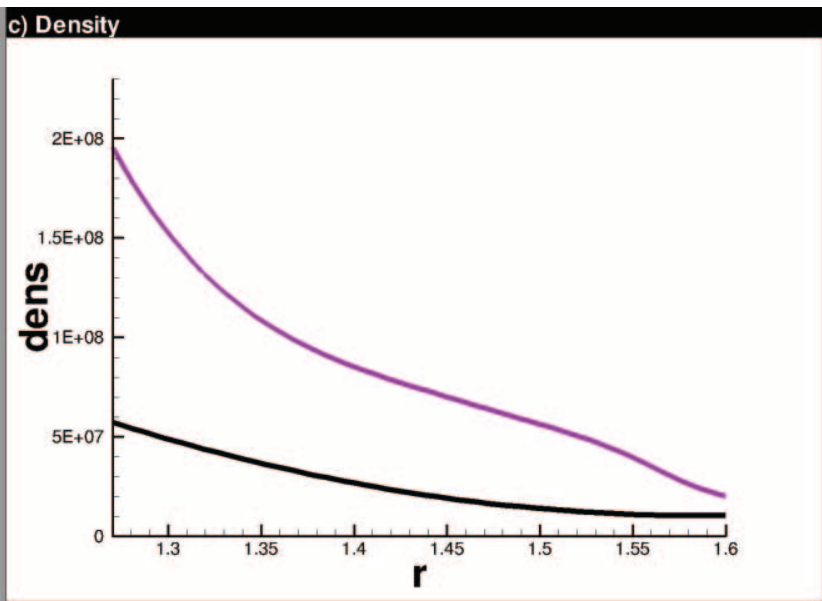

d) Magnetic field strength

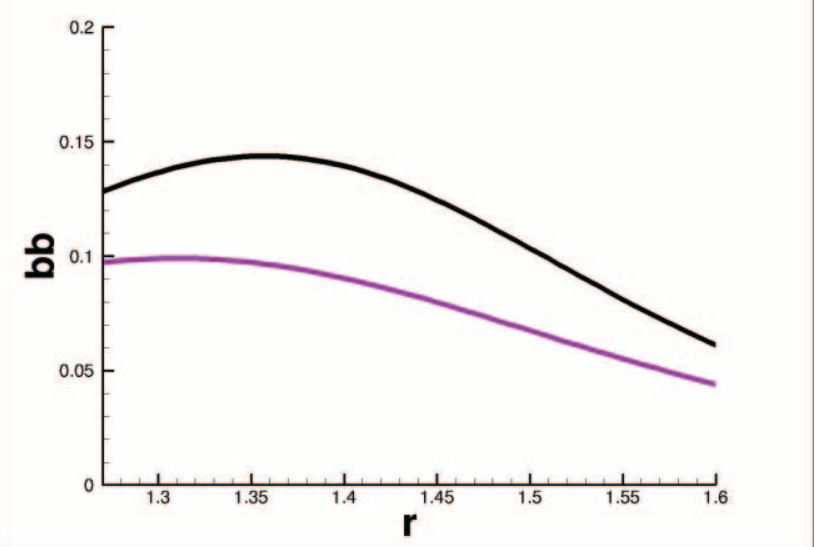

FIG. 15.-Axisymmetric flux rope model of Fan \& Gibson (2006). (a) Polar coordinates $(r-\theta)$ plot offield lines (white) overlaid on log(density) contours, demonstrating

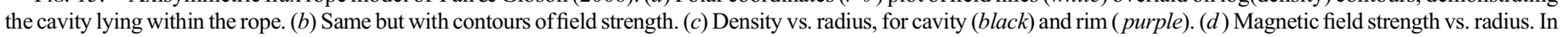
both $(a)$ and $(b)$, vertical line segments define "rim" ( purple) and "cavity" (black) regions as used in $(c)$ and $(d)$.

Van de Hulst density radial profiles determined in $\S 4$ to the hydrostatic density falloff as follows,

$$
\begin{gathered}
\log N_{e}(r)=\beta+\frac{\delta}{r}, \\
\beta=\log N_{e}\left(r_{0}\right)-\frac{1}{h_{0} r_{0}} ; \quad \delta=\frac{1}{h_{0}} .
\end{gathered}
$$

We obtain $\beta_{\text {rim }}=10.79 \pm 0.18 ; \delta_{\text {rim }}=8.60 \pm 0.22 ; \beta_{\text {cav }}=$ $12.00 \pm 0.20 ; \delta_{\text {cav }}=6.57 \pm 0.24$. This in turn implies scale heights at the coronal base $\left(r=r_{0}=1\right)$ of $h_{0_{\text {in }}}=0.116 \pm 0.003$; $h_{0_{\mathrm{cav}}}=0.152 \pm 0.005$ (in units of solar radii), and temperatures $T_{\text {rim }}=1.57 \pm 0.04 \times 10^{6} ; T_{\text {cav }}=2.05 \pm 0.07 \times 10^{6}$ (in units of kelvins). Thus, the flatter density profile in the cavity implies a larger scale height and correspondingly hotter (by $31 \% \pm 7 \%$ ) temperature relative to the rim.

How realistic is this sort of hydrostatic determination of temperature from white-light observations? Previous studies have compared hydrostatic temperatures to independent temperature diagnostics from emission-line ratios, with mixed results. The analysis of Gibson et al. (1999) focused on the core of a longitudinally symmetric streamer and found good agreement between white-light hydrostatic temperatures and emission-line temperatures. Guhathakurta et al. (1992) used eclipse observations in white light and found that, when averaged over all polar angles, the scale height temperatures agreed well with temperatures de- termined independently using emission-line ratios. Discrepancies arose, however, in particular near the boundaries of helmet streamers: this is to be expected since a radial profile at such a boundary would cross from streamer into surrounding coronal hole, violating the assumption of a homogenous region. More directly relevant to the analysis presented here, the Guhathakurta et al. (1992) analysis showed that one of the streamers possessed a prominence and surrounding cavity (Feature 11 in that paper). The scale-height temperature determined from the white-light eclipse observations implied that the cavity was hotter than its surrounding streamer, just as we have found. However, the temperature determined from emission-line ratios implied a decreased temperature in the cavity relative to the rim. This leads us now to consider whether there might be a reason other than an increased temperature and associated density scale height for the relatively flat density profile that we observe in our cavity.

\subsection{Magnetic Flux Rope Model}

Figure 15 illustrates magnetic and density structure for an axisymmetric model of a magnetic flux rope in magnetohydrodynamic equilibrium (Fan \& Gibson 2006). It demonstrates how such a flux rope would possess a cavity (Fig. 15a), corresponding to a region of particularly strong magnetic field strength (Fig. 15b). Adjusting for the somewhat larger size of this cavity, we select radial-cut subregions to identify as "rim" and "cavity" (Figs. 15a and $15 b$, purple and black vertical lines) that are equivalent to 


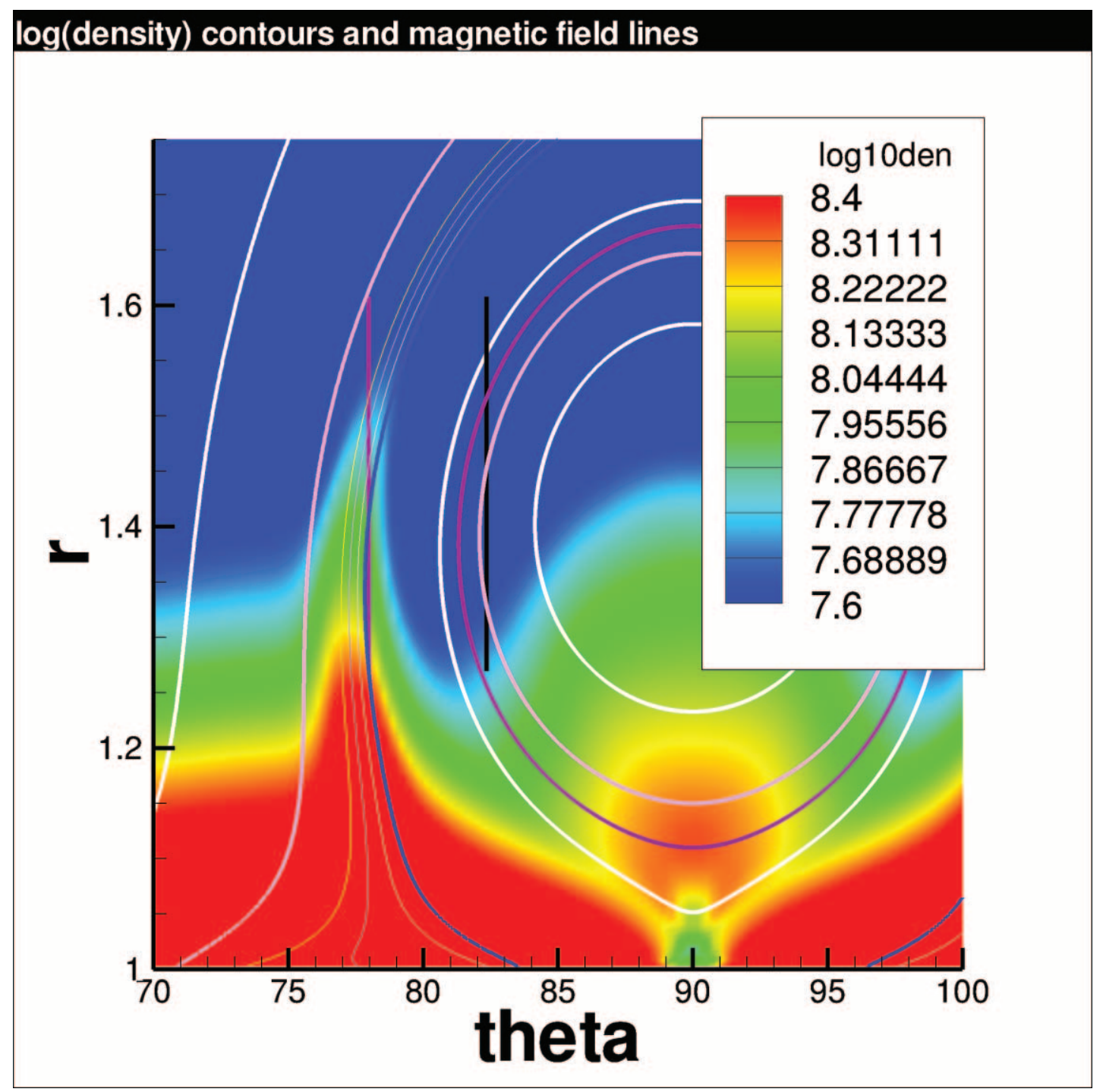

FIG. 16. - $\log$ (density) for axisymmetric flux rope model of Fan \& Gibson (2006) zoomed in and saturated to demonstrate the variation of the lower boundary density for field lines. Vertical line segments define "rim" ( purple) and "cavity" (black) regions as used in Fig. 15.

those chosen observationally (compare to Fig. 12, left). Figure $15 c$ shows density profiles of the model rim and cavity that are qualitatively very similar to what we have found observationally (Fig. 13, bottom); i.e., the rim density falls off more steeply than the cavity.

However, this flux rope model assumes a corona with constant temperature throughout. This strongly illustrates the perils of the hydrostatic temperature density diagnostic: if we were to apply it to the model density profiles, we would conclude that the cavity was hotter than the rim, in direct contradiction to the model specifications. Because the model is completely isothermal, variation in density must arise from differences in the boundary conditions on magnetic field lines. Figure 16 shows a zoomed-in view of field lines intersecting the rim and cavity radial cuts. The field lines intersecting most of the purple rim radial cut are rooted in a lower boundary that is dense relative to the outer region (see also Fig. 15a). At larger heights, the rim radial cut crosses through field lines that are rooted external to the high-density boundary under the rim, causing a steepened falloff in the density profile above $1.55 R_{\odot}$ seen in Figure $15 \mathrm{c}$. Below this height, however, the assumption of a uniform boundary condition would be reasonable, and the scale height tem- perature determined from the profile would be consistent with the isothermal model.

The black rim radial cut, on the other hand, intersects circular field lines that are disconnected from the lower boundary. Figure 16 demonstrates that because mass is accumulated in the concave "dips" of these field lines, the density at the lowest point of each circle (from which a hydrostatic falloff would originate) is larger than that of material at the same height but on the circular field line directly beneath it (although still smaller than that of material at that height on field lines connected to the rim lower boundary). The result is that for increasing height along the lower portion of the cavity radial cut (below approximately $1.4 R_{\odot}$ ) field lines of increasing relative boundary density are crossed, leading to a flatter density falloff. (Above $1.4 R_{\odot}$ [the axis of the rope] the slope is actually somewhat steepened as the radial cut crosses circular field lines of decreasing lower boundary density; however, the temperature of the simulation is such that all the field lines at these heights are in the flat tail of the exponential falloff, so the steepening is not apparent.) Thus, a scale height temperature based on the density along the flattened cavity radial cut would be overestimated because the assumption of a uniform lower boundary condition would be invalid. 


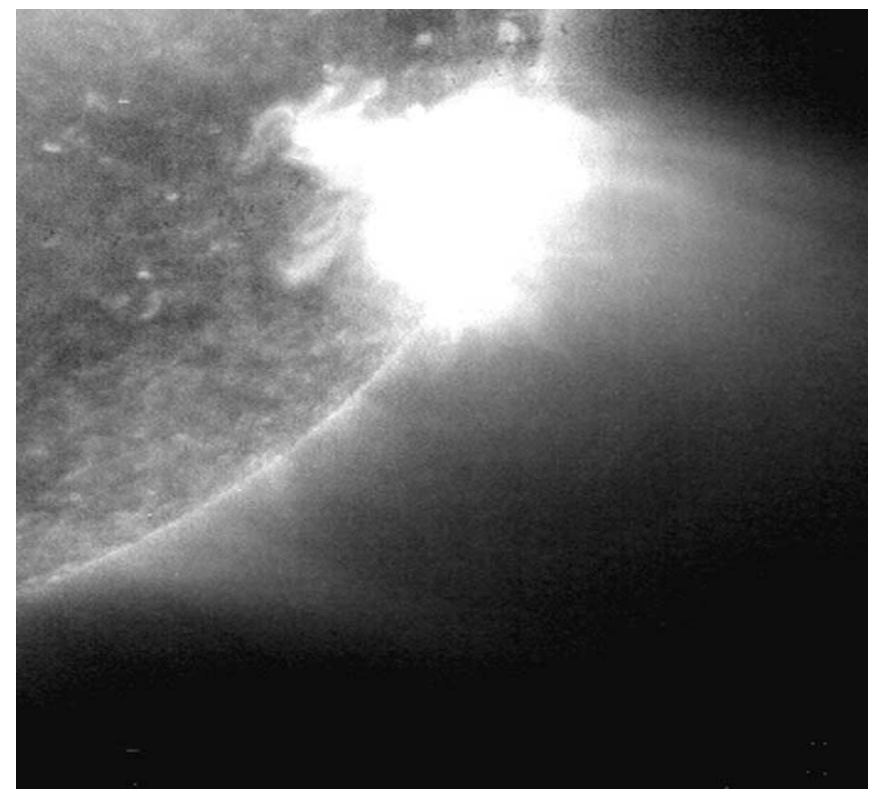

FIG. 17.-Cavity observed on 2006 January 27 at $01: 06$ UT in $284 \AA$ (SOHO EIT).

\subsection{Discussion}

Our conclusion based on the above analysis is that whitelight observations of a cavity are insufficient for establishing the cavity's temperature or magnetic structure. Two extremes can be seen to be potentially consistent with the density profiles determined from white-light observations. On the one hand, the cavity field lines might have essentially any orientation so long as they possess enhanced temperature and are rooted in a uniform lower boundary density (which must be depleted relative to the boundary of the rim). On the other hand, the "detached" field lines of a flux rope could explain the depletion and flatness of the cavity density profile relative to the rim without requiring increased temperature (and also yield a sharp circular boundary between cavity and rim; see, e.g., Fig. 15). In the physical corona, such flux rope field lines would not be completely detached circles as in the two-dimensional model described above, but rather winding field lines with prominence mass condensed in their dips serving to thermally disconnect them from the photosphere (Low 1996).
A logical future extension of the analysis presented here, then, would be to obtain independent information about a cavity's temperature or magnetic structure. New observations of coronal magnetic fields such as those being obtained by the HAO Coronal Multichannel Polarimeter (CoMP; Tomczyk et al. 2008) may be particularly valuable, as cavities are often visible in CoMP images (S. Tomczyk 2007, private communication). Alternatively, temperature might be obtained using emission-line observations of cavity plasma. Indeed, coronal cavities are often observed in emission, particularly in extreme ultraviolet (EUV) and soft X-ray (SXR; Hudson et al. 1999; Yurchyshyn 2002; Sterling \& Moore 2004; Maričić et al. 2004; Vršnak et al. 2004). Figure 17 shows the 2006 January 27 cavity as observed in EUV (284 $\AA$ ) by SOHO EIT. Geometrically speaking, such emission cavities have an advantage in avoiding contamination from the cavity rim because of the rapid falloff of emission with height: EUV emission is sensitive to density squared, as opposed to white light, which is sensitive to density. In fact, large emission cavities such as the one shown in Figure 17 do not have a clearly resolved cavity top. If the cavity rim (at the central polar angle) is not visible when it lies in the plane of the sky where limb-brightening effects would make it most pronounced, it should not contribute significantly to observed emission in the cavity as it bends into the line of sight. Whether or not cavity legs have an impact depends on how axisymmetric the cavity is. This can be determined by examining how many days the cavity is visible at the limb in emission as discussed above. For some cavities, evidence of axisymmetry can also be obtained from observations of the filament channel when it is on the disk. Unfortunately, cavities as large as our 2006 January case do not generally have well-resolved filament channels. However, cavities that are more compact sometimes have beautifully resolved polar crown filaments and filament channels, as Figure 18 demonstrates for the white-light cavity of Figure 1.

It is not difficult to find examples of EUV or SXR images of cavities that satisfy our model geometry. However, because instruments such as $\mathrm{SOHO}$ EIT have limited and wide passbands, they are insufficient to constrain the plasma temperature (Schmelz et al. 2007). An alternative is to use ratios of spectrally resolved emission lines to obtain temperatures (Fludra et al. 1999; it should be noted that even for spectral line ratios, departures from local thermal equilibrium [Edgar \& Esser 2000] and inhomogeneities along the line of sight [Aschwanden et al. 2000; Deforest 2007] need to be kept in mind when interpreting temperature diagnostics). Coronal spectrometers tend to have limited fields of

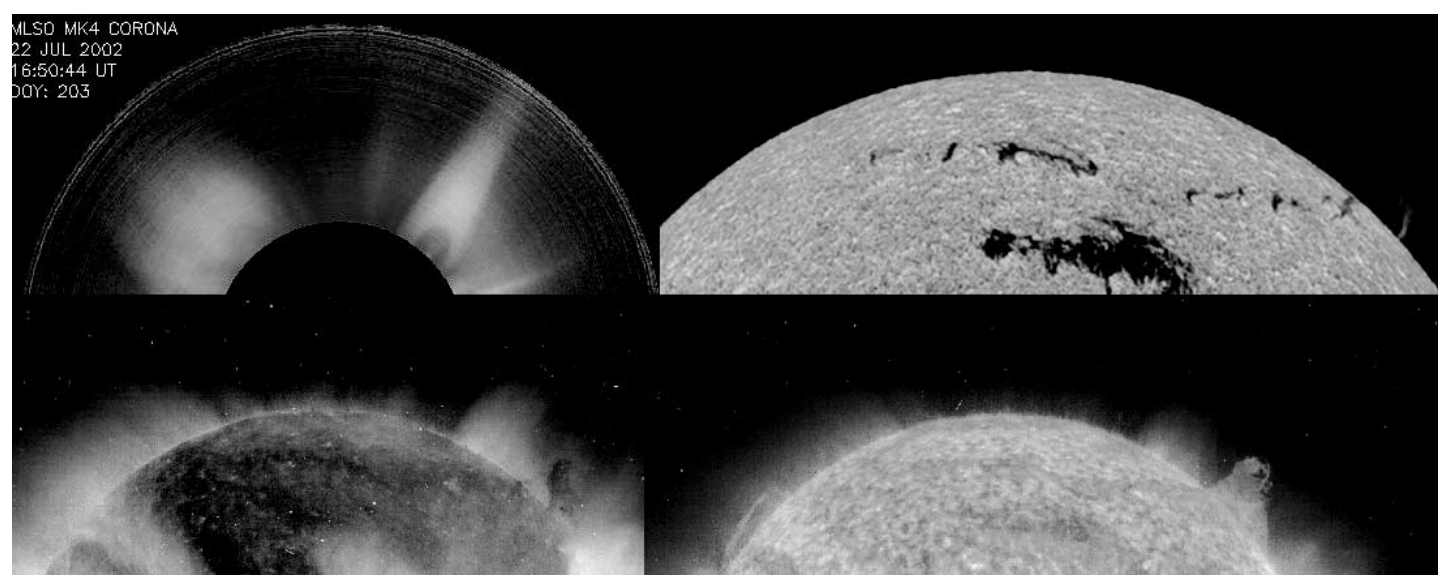

FIG. 18.-White-light cavity of 2002 July 22 as shown in Fig. 1, with $\mathrm{H} \alpha$ and EUV observations showing associated extended polar crown filament, filament channel, and emission cavity at the limb. Top left, white light ( $\mathrm{Mk} 4)$; top right, $\mathrm{H} \alpha$ (Big Bear Solar Observatory [BBSO]); bottom left, $284 \AA$ (SOHO EIT); bottom right, $304 \AA ̊$ (SOHO EIT). From Gibson et al. (2006). 
view and so must explicitly target cavities to obtain observations. We have shown in this paper that cavities have significant density, closer to that of streamers than of coronal holes, so it would be well worthwhile to obtain direct spectroscopic diagnostics of temperatures and velocities within cavities. If such diagnostics do not indicate a temperature gradient between rim and cavity, or indeed as appears to be the case for Guhathakurta et al. (1992) the emission-line observations indicate a cavity cooler than the rim, the thermal disconnection of a flux-rope-type model would be arguably the best candidate for explaining a flattened density falloff.

\section{CONCLUSIONS}

Despite the fact that coronal cavities are often obstructed by their surrounding helmet streamers, we have used our model to show that certain cavities (i.e., large cavities relatively close to the equator that maintain constant size and latitude for several days) are observed in white light with minimal contribution from surrounding material. By using a Van de Hulst inversion of $p B$ measurements, we have successfully measured the density of a cavity to a higher degree of accuracy than previously accomplished. We have determined that our cavity is roughly twice as dense as a coronal hole at low altitudes and roughly 5 times as dense at higher altitudes. The cavity density is depleted by about $40 \%$ compared to the cavity rim at low altitudes but shows very little or no depletion at high altitudes. We show that this flattened density profile could be due either to an enhanced cavity temperature relative to the rim, or a detached, magnetic flux rope topology, or conceivably a combination of the two effects. Emission-line diagnostics will be key to constraining and inspiring future cavity models that will no doubt need to include thermodynamic and dynamic effects explicitly, in order to obtain a three-dimensional, self-consistent description of the cavity plasma. In this manner, the physical properties of cavities, which are interesting both as MHD equilibrium states in the corona and as the potential source region of CMEs, may be probed.

We thank Joan Burkepile for internal review of this manuscript and for assistance with determining MK4 instrumental errors for the data used. We also thank David Elmore for assistance with determining MK4 errors. We thank Spiro Antiochos, Craig Deforest, Tom Holzer, Terry Kucera, and B. C. Low for helpful discussions. We would like to thank the National Science Foundation Research Experiences for Undergraduates (NSF-REU), the University of Colorado's Laboratory for Atmospheric and Space Physics (LASP), and the National Center for Atmospheric Research High Altitude Observatory (NCAR/HAO) for giving J. Fuller the opportunity to work at HAO as part of his summer 2007 NSF-REU program. We acknowledge Big Bear Solar Observatory and New Jersey Institute of Technology for the $\mathrm{H} \alpha$ data and $S O H O$ EIT for EUV data. SOHO is a mission of international collaboration between ESA and NASA. The Mauna Loa Solar Observatory is a facility of the National Center for Atmospheric Research and is sponsored by the National Science Foundation.
Aschwanden, M. J., Nightingale, R. W., \& Alexander, D. 2000, ApJ, 541, 1059 Billings, D. E. 1966, A Guide to the Solar Corona (New York: Academic) Deforest, C. 2007, ApJ, 661, 532

Edgar, R. J., \& Esser, R. 2000, ApJ, 539, 167

Elmore, D. F., Burkepile, J. T., Darnell, J. A., Lecinski, A. R., \& Stanger, A. L. 2003, Proc. SPIE, 4843, 66

Engvold, O. 1989, in Dynamics and Structures of Quiescent Prominences, ed. E. R. Priest (Dordrecht: Reidel), 47

Fan, Y., \& Gibson, S. E. 2006, ApJ, 641, L149

Fisher, R. R., \& Munro, R. H. 1984, ApJ, 280, 428

Fisher, R. R., \& Poland, A. I. 1981, ApJ, 246, 1004

Fludra, A., Del Zanna, G., Alexander, D., \& Bromage, B. J. I. 1999, J. Geophys. Res., 104, 9709

Gibson, S. E., Fludra, A., Bagenal, F., Biesecker, D., Del Zanna, G., \& Bromage, B. 1999, J. Geophys. Res., 104, 9691

Gibson, S. E., Foster, D., Burkepile, J., de Toma, G., \& A., S. 2006, ApJ, 641, 590

Guhathakurta, M., Fludra, A., Gibson, S. E., Biesecker, D., \& Fisher, R. 1999, J. Geophys. Res., 104, 9801

Guhathakurta, M., Rottman, G. J., Fisher, R. R., Orrall, F. Q., \& Altrock, R. C. 1992, ApJ, 388, 633

Habbal, S. R., Esser, R., \& Arndt, M. B. 1993, ApJ, 413, 435

Hudson, H. S., Acton, L. W., Harvey, K. A., \& McKenzie, D. M. 1999, ApJ, 513,83

\section{REFERENCES}

Hundhausen, A. J. 1993, J. Geophys. Res., 98, 13177

1999 , in The Many Faces of the Sun: a Summary of the Results of NASA's Solar Maximum Mission, ed. K. T. Strong et al. (New York: Springer), 143

Illing, R. M., \& Hundhausen, A. J. 1986, J. Geophys. Res., 91, 10951

Kundu, M. R., Fuerst, E., Hirth, W., \& Butz, M. 1978, A\&A, 62, 431

Low, B. C. 1996, Sol. Phys., 167, 217

Maričić, D., Vršnak, B., Stanger, A. L., \& Veronig, A. 2004, Sol. Phys., 225, 337

Marque, C. 2004, ApJ, 602, 1037

Priest, E. R. 1982, Solar Magnetohydrodynamics (Dordrecht: Reidel)

Saito, K., \& Hyder, C. 1968, Sol. Phys., 5, 61

Saito, K., \& Tandberg-Hanssen, E. 1973, Sol. Phys., 31, 105

Schmelz, J. T., Kashyap, V. L., \& Weber, M. A. 2007, ApJ, 660, L157

Sterling, A. C., \& Moore, R. L. 2004, ApJ, 613, 1221

Straka, R. M., Papagiannis, M. D., \& Kogut, J. A. 1975, Sol. Phys., 45, 131 Tandberg-Hanssen, E. 1974, Solar Prominences (Dordrecht: Reidel)

. 1995, The Nature of Solar Prominences (Dordrecht: Kluwer)

Tomczyk, S., et al. 2008, Sol. Phys., 247, 411

Van de Hulst, H. C. 1950, Bull. Astron. Inst. Netherlands, 11, 135

Vršnak, B., Maričić, D., Stanger, A. L., \& Veronig, A. 2004, Sol. Phys., 225, 355

Wiik, J. E., Schmieder, B., \& Noens, J. C. 1994, Sol. Phys., 149, 51

Yurchyshyn, V. B. 2002, ApJ, 576, 493 